\title{
A systematic review and meta-analysis of the potential non-human animal reservoirs and arthropod vectors of the Mayaro virus
}

Michael Celone $^{1^{*}}$, Bernard Okech ${ }^{1}$, Barbara A. Han ${ }^{2}$, Brett M. Forshey ${ }^{3}$, Assaf Anyamba ${ }^{4}$, James Dunford ${ }^{1}$, George Rutherford $^{5}$, Neida K. Mita Mendoza ${ }^{6}$, Elizabet Lilia Estallo ${ }^{7}$, Ricardo Khouri ${ }^{8}$, Isadora Cristina de Siqueira ${ }^{8}$, Simon

Pollett ${ }^{9,10}$

${ }^{1}$ Uniformed Services University of the Health Sciences, F. Edward Hébert School of Medicine, Department of Preventive Medicine \& Biostatistics, Bethesda, Maryland

${ }^{2}$ Cary Institute of Ecosystem Studies, NY, USA

${ }^{3}$ Armed Forces Health Surveillance Division, Silver Spring, MD, USA

${ }^{4}$ University Space Research Association \& NASA/Goddard Space Flight Center, Biospheric Sciences Laboratory, Greenbelt, MD, USA

${ }^{5}$ Institute for Global Health Sciences, University of California, San Francisco, San Francisco, California, USA

${ }^{6}$ New York State Department of Health, NY, USA

${ }^{7}$ Instituto de Investigaciones Biológicas y Tecnológicas (IIByT) CONICET-Universidad Nacional de Córdoba. Centro de Investigaciones Entomológicas de Córdoba, Córdoba, Argentina (https://orcid.org/0000-0002-6723-6929)

${ }^{8}$ Instituto Gonçalo Moniz-Fiocruz, R. Waldemar Falcão, Salvador-BA, Brazil

${ }^{9}$ Infectious Disease Clinical Research Program, Department of Preventive Medicine and Biostatistics, Uniformed Services University of the Health Sciences, Bethesda, MD, USA

${ }^{10}$ Henry M. Jackson Foundation for the Advancement of Military Medicine, Inc., Bethesda, MD, USA

*Corresponding author

Michael.celone@usuhs.edu (MC) 


\section{Abstract}

Improving our understanding of Mayaro virus (MAYV) ecology is critical to guide surveillance and risk assessment. We conducted a PRISMA-adherent systematic review of the published and grey literature to identify potential arthropod vectors and non-human animal reservoirs of MAYV. We searched PubMed, Embase, Web of Science, SciELO and greyliterature sources including PAHO databases and dissertation repositories. Studies were included if they assessed MAYV virological/immunological measured occurrence in field-caught, domestic, or sentinel animals or in field-caught arthropods. We conducted an animal seroprevalence meta-analysis using a random effects model. We compiled granular georeferenced maps of non-human MAYV occurrence and graded the quality of the studies using a customized framework. Overall, 57 studies were eligible out of 1523 screened, published between the years 1961 and 2020. Seventeen studies reported MAYV positivity in wild mammals, birds, or reptiles and five studies reported MAYV positivity in domestic animals. MAYV positivity was reported in 12 orders of wild-caught vertebrates, most frequently in the orders Charadriiformes and Primate. Sixteen studies detected MAYV in wild-caught mosquito genera including Haemagogus, Aedes, Culex, Psorophora, Coquillettidia, and Sabethes. Vertebrate animals or arthropods with MAYV were detected in Brazil, Panama, Peru, French Guiana, Colombia, Trinidad, Venezuela, Argentina, and Paraguay. Among non-human vertebrates, the Primate order had the highest pooled prevalence (PP) at $13.1 \%$ (95\% CI: 4.3$25.1 \%$ ). From the three most studied primate genera we found the highest prevalence was in Alouatta (PP: 32.2\%, 95\% CI: 0.0-79.2\%), followed by Callithrix (PP: 17.8\%, 95\% CI: 8.628.5\%), and Cebus/Sapajus (PP: 3.7\%, 95\% CI: 0.0-11.1\%). We further found that MAYV occurs in a wide range of vectors beyond Haemagogus spp. The quality of evidence behind these findings was variable and prompts calls for standardization of reporting of arbovirus occurrence. These findings support further risk emergence prediction, guide field surveillance efforts, and prompt further in-vivo studies to better define the ecological drivers of MAYV maintenance and potential for emergence.

\section{Author Summary}

Mayaro virus (MAYV) is an emerging tropical public health threat in the Americas. We conducted a georeferenced, quality-graded systematic review to evaluate the current evidence regarding MAYV occurrence in non-human vertebrates and arthropods. Overall, 57 studies were eligible out of 1523 screened, published between the years 1961 and 2020. Seventeen studies reported MAYV positivity in wild mammals, birds, or reptiles and five studies reported MAYV positivity in domestic animals. MAYV positivity was reported in 12 orders of wild-caught vertebrates, most frequently in the orders Charadriiformes and Primate. Our systematic review identified 12 orders of wild-caught vertebrates and seven mosquito genera with evidence of MAYV occurrence. Primates had the highest pooled MAYV prevalence according to a seroprevalence meta-analysis. The graded quality of evidence behind these findings was variable and prompts calls for standardization of reporting of MAYV and perhaps other emerging arbovirus occurrence in animals and vectors. This study provides important information for public health authorities and disease ecologists concerned with the growing threat of MAYV in Latin America. Our analysis provides a foundation for future laboratory and field studies focused on the MAYV transmission cycle. 


\section{Introduction}

First detected in Trinidad in 1954 [1], Mayaro virus (MAYV) is a zoonotic Alphavirus

85 that is endemic in several Latin American countries. Like Chikungunya virus (CHIKV), MAYV

86 may cause complications such as debilitating arthralgia but often presents with a non-specific

87 constellation of symptoms and signs that may be clinically indistinguishable from other vector

88 borne diseases such as dengue or Zika [2]. There is no current licensed vaccine or antiviral

89 treatment for MAYV infections, and the current standard of clinical treatment is supportive care

90 only $[2,3]$.

91 MAYV has caused periodic outbreaks in humans in Brazil [4, 5], Bolivia [6], and

92 Venezuela [7], while surveillance studies and serological surveys have detected MAYV in

93 humans in several countries throughout the Americas including Peru [8], Suriname [9], Mexico

94 [10], Colombia [11], French Guiana [12], and Haiti [13]. These findings demonstrate widespread

95 circulation of the virus throughout the region. A recent 2019 epidemiological alert by the Pan

96 American Health Association (PAHO) has emphasized the need for increased awareness of and

97 extended surveillance for this emerging virus in the Americas [3]. However, the precise areas of

98 risk from MAYV throughout the Americas remain unclear. Understanding the ecology and

99 distribution of MAYV remains a major obstacle in predicting areas that are at high risk of

100 transmission to humans and domestic animals.

101 Current evidence suggests that MAYV is maintained in nature through a sylvatic

102 transmission cycle involving mosquito vectors and non-human animal reservoirs. Therefore,

103 human MAYV cases reported to date likely represent direct sylvatic spillovers. Residing near

104 forested areas [12] and hunting in the rainforest [14] have been identified as risk factors for 
MAYV infection in humans, highlighting the importance of the sylvatic transmission cycle and

106 the potential for spillover events.

108 transmission is an important step in delineating the human populations at greatest risk. The

109 spillover of MAYV into humans represents a complex interaction of processes involving the

110 density and distribution of reservoirs and vectors, as well as the prevalence and intensity of

111 infection among reservoirs [15].

Identifying the non-human vertebrates that may serve as MAYV reservoirs is a difficult

113 task due to a myriad of issues including, but not limited to, the challenges associated with

114 establishing evidence of infection in wild animal populations $[16,17]$. High seroprevalence of a

115 pathogen in an animal population does not necessarily implicate a given host as an efficient

116 reservoir; conversely, low seroprevalence at a single point in time cannot definitively rule out an

117 animal as a reservoir [17]. Due to the relatively short viremia of MAYV (approximately 3-10

118 days) molecular assays may be unsuccessful in detecting virus [18], necessitating the use of

119 serological assays such as hemagglutination-inhibition (HI) assays, enzyme-linked

120 immunosorbent assays (ELISA), or plaque-reduction neutralization tests (NT).

121 Several studies have been conducted to clarify the precise vertebrate hosts that may serve

122 as MAYV reservoirs. High seroprevalence among non-human primates (NHPs) in Brazil [19],

123 French Guiana [12], and Panama [20] provides evidence that NHPs may play an important role

124 in the MAYV transmission cycle. MAYV antibodies have also been detected in mammals

125 including rodents and marsupials [21] as well as several avian species [19]. Unfortunately, there

126 is significant heterogeneity in the study methods used to identify potential MAYV reservoirs and 
127 there remains a high level of uncertainty surrounding the role of various non-human vertebrate

128 species in the MAYV transmission cycle.

129 Studies have also been conducted in wild-caught mosquito populations as well as in

130 controlled laboratory conditions in order to identify potential arthropod vectors of MAYV. One

131 study in Brazil [19] suggested that the canopy-dwelling Haemagogus janthinomys mosquito is an

132 important vector of MAYV. Additional mosquito species including Aedes aegypti, Ae.

133 albopictus, and several anopheline species have been shown to be competent vectors in

134 laboratory settings [22-24], posing a potential but as yet theoretical risk of urban MAYV cycles.

135 The occurrence of MAYV in the city of Manaus has also led to concerns about the involvement

136 of Aedes mosquitoes in a MAYV urban transmission cycle [25].

137 Although many non-human vertebrate animals and arthropod species have been proposed

138 as capable MAYV reservoirs or vectors, our understanding of the MAYV transmission cycle and

139 ecology remains limited. Collating and evaluating the current evidence regarding the potential

140 MAYV reservoirs and vectors are important steps in characterizing MAYV transmission ecology

141 and identifying the communities at greatest risk for MAYV outbreaks. Therefore, the goal of this

142 systematic review is to evaluate the current evidence regarding MAYV occurrence in non-human

143 vertebrates and arthropods. We present here the first structured evaluation of the potential vector

144 and non-human reservoir range of MAYV, including the development of custom criteria for

145 grading the quality of evidence of arbovirus occurrence in invertebrate and vertebrate non-human 146 hosts. 


\section{Methods}

This systematic review and meta-analysis were conducted according to the PRISMA

1492020 Checklist [26] (see S1 Table). A protocol was developed but was not uploaded to

150 PROSPERO.

\section{Information Sources}

We conducted a systematic review of original research articles, reports, and dissertations

153 that attempted to identify potential non-human animal reservoirs or arthropod vectors of MAYV.

154 We first searched Embase, Web of Science, PubMed, and SciELO databases for English,

155 Spanish, and Portuguese language articles published between 1954 (the year MAYV was first

156 isolated) and March 21, 2020. We searched all databases using the highly sensitive search term

157 "Mayaro". A PubMed alert using the search term "Mayaro" was also set to capture any

158 additional studies that were published between the initial search and May 2021. This database

159 search was extended using bioRxiv (https://www.biorxiv.org/) and medRxiv

160 (https://www.medrxiv.org/) pre-print databases. We complemented these database search results

161 with 'grey literature,' including hand-searched bibliographies of MAYV review articles

162 (including systematic reviews), dissertations from several Brazilian university repositories, the

163 Pan American Health Organization (PAHO) Institutional Repository for Information Sharing

164 database (iris.paho.org), the GIDEON database (https://www.gideononline.com/), and GenBank

165 [27] (https://www.ncbi.nlm.nih.gov/genbank/). In addition, we searched conference handbooks

166 that are available online (2004-2019) from the American Society of Tropical Medicine and

167 Hygiene (https://www.astmh.org/annual-meeting/past-meetings). 


\section{Eligibility Criteria}

We included studies that evaluated past or current MAYV infection in non-human

170 vertebrates using methods including virus isolation, molecular detection, and serosurveys. We

171 also included studies that screened arthropods for MAYV using virus isolation and molecular

172 detection. Original research studies were considered for eligibility if they assessed MAYV

173 positivity in field-caught, captive, or sentinel non-human vertebrates or field-caught arthropods.

174 Studies that met any of the following exclusion criteria were not included: studies involving only

175 humans; studies not reporting original data (e.g., review articles, perspective pieces, editorials,

176 recommendations, and guidelines); duplicate studies; in vitro studies such as vector cell-line or

177 mammal cell line experiments; laboratory-based vector competence studies that did not explicitly

178 demonstrate the detection of MAYV in a wild-caught vector; in-vivo lab-reared animal studies or

179 any laboratory-based study that experimentally inoculated an animal to test theoretical reservoir

180 status.

181 Selection process

All articles were organized using EndNote software version X9 (Clarivate, Philadelphia,

183 Pennsylvania, USA), and data were abstracted into a Microsoft Excel table. Two reviewers

184 independently screened all titles and abstracts to determine articles that could immediately be

185 excluded and articles that should be included in the second stage of review. Results were

186 compared to reconcile any differences between the two reviewers. The first and second reviewers

187 then independently read the full text of potentially eligible articles identified through screening

188 and selected the articles that were candidates for inclusion in the study. Results were compared

189 to reconcile any differences between the two reviewers. A third-party reviewer adjudicated when

190 consensus was not reached between the two reviewers during the first or second stage review. 
191 From those studies deemed eligible, data were extracted from articles by one reviewer using the

192 data abstraction tool in Microsoft Excel.

\section{Data abstraction}

Relevant information was abstracted by one reviewer in an Excel sheet. Information for

195 each article was abstracted across several domains including publication details (author and

196 affiliation, study title, study funding), study methods (date and location of study, study design,

197 laboratory methods to assess MAYV positivity), and study results (sample size, taxonomic

198 classification, proportion of animals testing positive for MAYV, location of

199 vertebrates/arthropods testing positive for MAYV). A second reviewer randomly selected and

200 reviewed five articles for review to validate the data abstraction process.

\section{Grading quality of evidence}

We developed a customized grading system to assess the quality of each study included

203 in our review. Several published studies have employed a similar grading system to assess

204 evidence quality of included articles [28-30]. We assigned each study in our systematic review a

205 grade for each of four quality items: clarity of research question/objective (Was the research

206 question/objective clearly described and stated?); description of study methods (Were the study

207 methods presented in a reproducible way?); description of sampling methods (Was the sampling

208 method described in detail?); and validity of diagnostic tests (Was MAYV positivity measured in

209 a valid way?). For each quality item, eligible studies were assigned a score of 3 (strong

210 evidence), 2 (moderate evidence), 1 (weak evidence), or unable to judge. Studies were deemed

211 unable to judge if the information provided was insufficient to assign quality scores (e.g., a

212 single GenBank entry or conference abstract). 
A score of 3 was assigned for the description of sampling methods item if authors

214 thoroughly described the type of trap used, the habitats in which traps were set, how often traps

215 were checked, and the results of trapping (i.e., were animals reported to the species level). For

216 studies that assessed MAYV in vertebrate animals, a score of 3 was assigned for the validity of

217 diagnostic tests item if MAYV positivity was assessed using RT-PCR, viral culture, or high-

218 specificity serological method (i.e., plaque reduction NT); a score of 2 was assigned if MAYV

219 positivity was assessed using non-specific serological assay (i.e., HI and ELISA); and a score of

2201 was assigned if MAYV positivity was based on presumptive exposure only with no

221 confirmatory assay. For studies that assessed MAYV in arthropods, a score of 3 was assigned for

222 this item if MAYV positivity was assessed using viral culture; a score of 2 was assigned if

223 MAYV positivity was assessed using RT-PCR or metagenomics; and a score of 1 was assigned

224 if MAYV positivity was based on presumptive exposure only with no confirmatory assay. A

225 score of "NA" was assigned for the validity of diagnostic tests item if studies did not detect

226 MAYV positivity in any animal or arthropod samples.

227 Quality review scores were recorded in two different Excel documents for animal

228 reservoir studies and arthropod vector studies, respectively. Two reviewers independently graded

229 the evidence quality for each study and results were compared to reconcile any differences

230 between the two reviewers. A third-party reviewer adjudicated if consensus was not reached

231 between the two reviewers. 


\section{Data analysis}

\section{Descriptive Analysis}

Descriptive statistics were presented by species for potential animal reservoirs showing

235 the total sample size, proportion infected, and locations of infected animals. Descriptive

236 statistics were presented by species for potential arthropod vectors showing the total sample size

237 and total pools tested for virus (if applicable), the number of MAYV isolates or PCR-positive

238 pools, and locations of infected arthropods. Maps were developed using ArcGIS software [31] to

239 display the geographic distribution of MAYV-positive animals and vectors.

\section{Pooled Analysis}

Due to the heterogeneity of study designs and outcome measurements, a quantitative

242 meta-analysis across all eligible studies was not possible. Instead, we conducted a seroprevalence

243 meta-analysis using the studies that reported MAYV seroprevalence (i.e., using serological

244 methods including HI, ELISA, or NT) in non-human vertebrate animals. Pooled prevalence

245 estimates were stratified by taxonomic order and an additional analysis was conducted among

246 the various Primate genera. Orders were excluded from the analysis if the total sample size was

247 less than 10 or if no MAYV-positive samples were reported within that order. Pooled

248 seroprevalence was first calculated based on all available data, regardless of test method. This

249 included the samples that tested MAYV-positive based on HI alone (when no confirmatory assay

250 was performed) as well as the samples that were confirmed positive by an NT. Only monotypic

251 reactions to MAYV were included in the meta-analysis in the absence of confirmatory NT. A

252 sensitivity analysis was then conducted using only the MAYV-positive samples that were

253 confirmed using NT. Positive samples that were based on HI alone (without confirmatory NT) 
254 were excluded from this analysis, although all MAYV-negative samples were retained. This

255 sensitivity analysis was conducted to account for the low specificity of HI compared to NT [32]

256 and provided a more conservative estimate of seroprevalence.

257 Due to the substantial differences across studies including sample size, study design,

258 species sampling methods, and geographical location, a random effects model was used for

259 analysis [33, 34]. The Freeman-Tukey double-arcsine transformation was implemented to

260 calculate a proportion, based on the recommendation of Barendregt et al. [35]. A sensitivity

261 analysis was conducted using a generalized linear mixed model (GLMM) with a logit

262 transformation, due to the potential for misleading results with the double-arccosine

263 transformation [36, 37]. Measures of variance $\left(\tau^{2}\right)$, heterogeneity $\left(I^{2}\right)$, and statistical significance

264 are presented for each random effects model. An additional sensitivity analysis was conducted

265 using a fixed effects model. Results of sensitivity analyses are presented in the Supplementary

266 materials.

267 The $I^{2}$ statistic measures inconsistency across study results and is calculated as $I^{2}=100 \%$

$268 \mathrm{x}(\mathrm{Q}-\mathrm{df}) / \mathrm{Q}[38]$. The $I^{2}$ statistic ranges between $0 \%$ and $100 \%$, where a value of $0 \%$ represents

269 no heterogeneity and larger values represent increased heterogeneity. Animal seroprevalence

270 estimates with $95 \%$ confidence intervals (CIs) weighted by sample size are presented as forest

271 plots. All analyses were conducted using the 'meta' package in R statistical software version

272 4.0.2 (R Project for Statistical Computing, Vienna, Austria) [39, 40].

\section{Estimation of bias}

275 studies or more. Bias was assessed using funnel plots and tests for funnel plot asymmetry based 
on methods proposed by Egger [41]. If the Egger's test revealed bias, the Trim and Fill technique

277 was used to estimate the effect of missing studies on the outcomes of the meta-analysis [42].

\section{Georeferencing of MAYV occurrence}

All available location information from each confirmed MAYV infection (animal and mosquito) was extracted from each article and georeferenced based on methods that have been

281 described previously [43, 44]. Each occurrence of MAYV was designated as either a point or

282 polygon location according to the spatial resolution provided in the study. When specific latitude

283 and longitude coordinates were provided, they were verified in GoogleMaps and designated as a

284 point location. If a neighborhood, town, village, or small city was explicitly mentioned in the

285 article and fell within a $5 \times 5 \mathrm{~km}$ grid cell, it was designated as a point location and its centroid

286 coordinates were recorded. For studies that report a less precise spatial resolution such as states

287 or counties, first level (ADM1) or second level (ADM2) administrative divisions were recorded

288 as polygons. If the size of a specific named location was greater than a $5 \times 5 \mathrm{~km}$ grid cell the

289 occurrence was assigned to a custom polygon created in ArcGIS that encompassed the extent of

290 that location. If place names were duplicated (i.e., the ADM1 and ADM2 units had the same

291 name), the coarsest spatial resolution was used. Country shapefiles were accessed through the

292 geoBoundaries Global Administrative Database [45].

\section{Results}

\section{General Findings}

We identified a total of 57 research items that met our eligibility criteria out of 1523

296 research items screened, including 46 research articles, seven dissertations, two GenBank entries,

297 one laboratory report, and one abstract (see Table 1 for a full list of eligible items and citations). 
298 Thirty-nine $(68 \%)$ of the included items assessed MAYV infection in non-human vertebrates

299 while $29(51 \%)$ items assessed MAYV infection in arthropods. Of the 57 eligible items, 24

$300(42 \%)$ were included in the vertebrate seroprevalence meta-analysis, and the remaining items

301 were only included in the qualitative analysis. A flow chart describing the article search and

302 selection process is presented in Fig 1. Five articles were identified that met the inclusion criteria

303 but were deemed to be reporting the same data as other included articles. These include de

304 Thoisy et al., (2001) [46] and Talarmin et al., (1998) [12] (both reporting the same data as de

305 Thoisy et al., (2003) [21]), Aitken et al., (1960) [47] (reporting the same data as Aitken et al.,

306 (1969) [48]), Batista et al., 2013 [49] (reporting the same data as Paulo et al., (2015) [50]), and

307 Woodall (1967) [51] (reporting the same data as Taylor, (1967) [52]). These articles were

308 excluded from this systematic review.

309 Table 1. Eligible Study Characteristics

\begin{tabular}{|l|l|l|l|l|l|}
\hline Reference & $\begin{array}{l}\text { Study } \\
\text { Period }\end{array}$ & Country & $\begin{array}{l}\text { Arthropods Tested } \\
(\mathbf{n})\end{array}$ & $\begin{array}{l}\text { Vertebrate } \\
\text { non-human } \\
\text { animals tested } \\
(\mathbf{n})^{\mathbf{a}}\end{array}$ & $\begin{array}{l}\text { MAYV } \\
\text { infection } \\
\text { reported }\end{array}$ \\
\hline Aitken, 1969 [48] & $1953-1963$ & Trinidad & $1,568,439$ & --- & Yes \\
\hline Araujo, 2003 [53] & 2002 & Brazil & --- & 555 & Yes \\
\hline Araujo, 2004 [54] & 2003 & Brazil & --- & 202 & No \\
\hline Araujo, 2004b [55] & 2003 & Brazil & --- & 495 & Yes \\
\hline Araujo, 2012 [56] & $2007-2008$ & Brazil & --- & 95 & Yes \\
\hline Araujo, 2012b [57] & 2009 & Brazil & --- & 102 & Yes \\
\hline Azevedo, 2009 [58] & 2008 & Brazil & 832 & --- & Yes \\
\hline Batista, 2012 [59] & 2010 & Brazil & 122 & 65 & Yes \\
\hline Calisher, 1974 [60] & 1967 & USA & --- & 1,300 & Yes \\
\hline Carrera, 2020 [61] & 2017 & Panama & 113 & --- & No \\
\hline Casseb, 2010 [62] & 2009 & Brazil & --- & 2191 & Yes \\
\hline Casseb, 2016 [63] & 2009 & Brazil & --- & 753 & Yes \\
\hline Catenacci, 2017 [64] & $2006-2014$ & Brazil & 239 & 142 & Yes \\
\hline Cruz, 2009 [65] & $2006-2008$ & Brazil & --- & 85 & No \\
\hline Degallier, 1992 [66] & $1974-1988$ & Brazil & $2,005,069$ & 6,248 & Yes \\
\hline De Thoisy, 2003 [21] & $1994-1995$ & French Guiana & --- & 579 & Yes \\
\hline Diaz, 2007 [67] & 1994 & $\begin{array}{l}\text { Argentina, } \\
\text { Paraguay }\end{array}$ & --- & 90 & No \\
\hline Esposito, 2015 [68] & 1960 & Brazil & NA & & Yes \\
\hline Ferreira, 2020 [69] & $2017-2018$ & Brazil & 10,569 & --- & Yes \\
\hline Galindo, 1966 [70] & $1959-1962$ & Panama & 377,492 & --- & Yes \\
\hline Galindo, 1967 [71] & 1966 & Panama & 11,829 & 2,444 & Yes \\
\hline
\end{tabular}




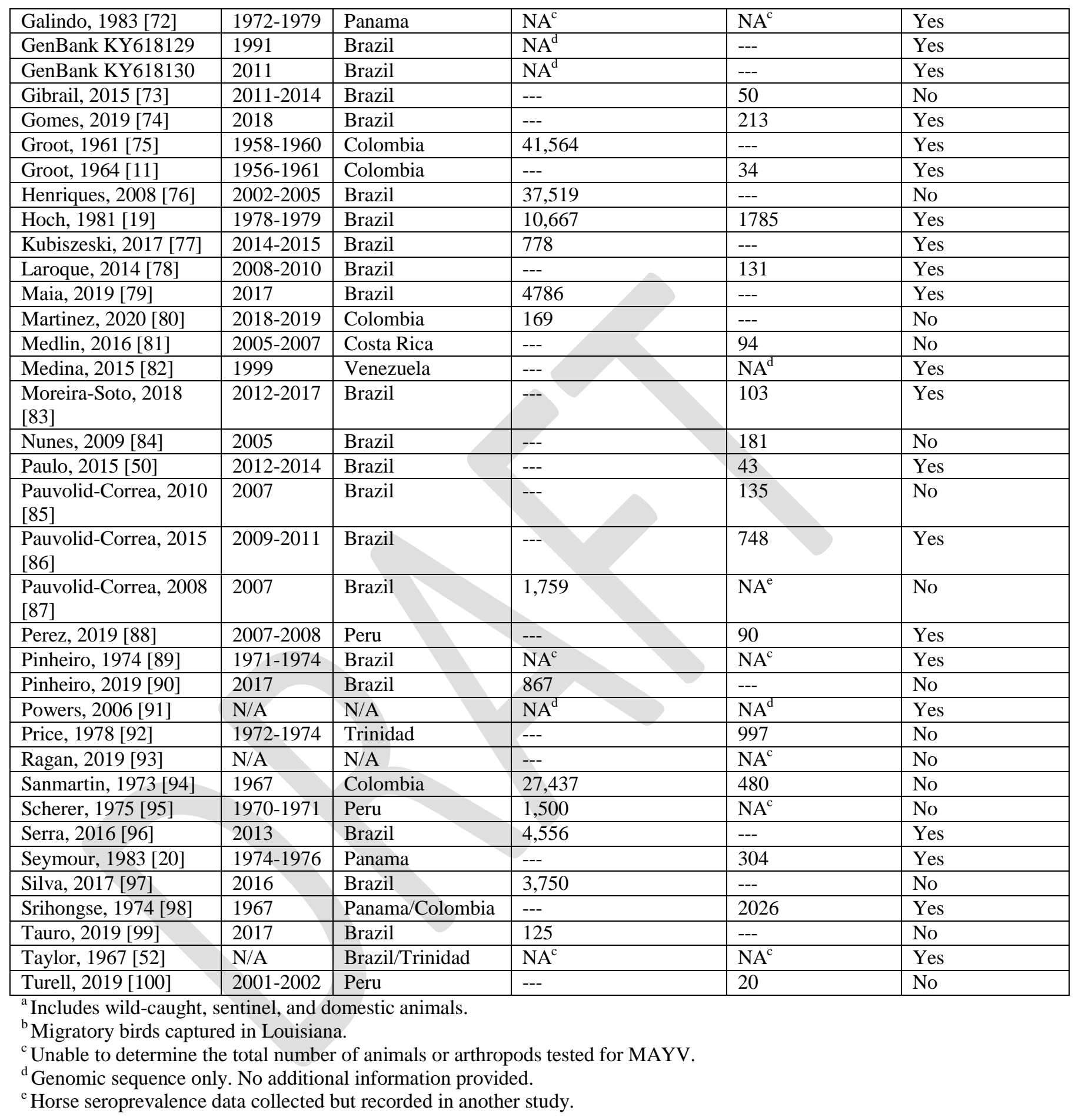

317 Fig 1. Flow diagram for search and selection of articles 
$320(\mathrm{n}=1)$, Costa Rica $(\mathrm{n}=1)$, and the United States of America $(\mathrm{n}=1)$. Several studies reported data

321 from multiple countries including Argentina/Paraguay (n=1), Panama/Colombia ( $\mathrm{n}=1)$, and

322 Brazil/Trinidad and Tobago $(n=1)$. The majority of studies were conducted after the year 2000

$323(\mathrm{n}=33)$, although some studies were conducted between 1950-1969 $(\mathrm{n}=9), 1970-1989(\mathrm{n}=8)$, or

324 1990-1999 (n=4). Quality scores for all included studies are reported in Table 2.

\section{Table 2. Quality Review Scores}

\begin{tabular}{|c|c|c|c|c|c|c|c|c|}
\hline & \multicolumn{4}{|c|}{ Vertebrate animals } & \multicolumn{4}{|c|}{ Arthropods } \\
\hline & $\begin{array}{l}\text { Research } \\
\text { question }\end{array}$ & $\begin{array}{l}\text { Study } \\
\text { methods }\end{array}$ & $\begin{array}{l}\text { Sampling } \\
\text { method }\end{array}$ & $\begin{array}{l}\text { MAYV+ test } \\
\text { method }^{\text {a }}\end{array}$ & $\begin{array}{l}\text { Research } \\
\text { question }\end{array}$ & $\begin{array}{l}\text { Study } \\
\text { methods }\end{array}$ & $\begin{array}{l}\text { Sampling } \\
\text { method }\end{array}$ & $\begin{array}{l}\text { MAYV+ } \\
\text { test method }\end{array}$ \\
\hline Aitken, 1969 [48] & --- & --- & --- & --- & 3 & 2 & 2 & 3 \\
\hline Araujo, 2003 [53] & 3 & 3 & 2 & 2 & --- & --- & --- & --- \\
\hline Araujo, 2004 [54] & 3 & 3 & 3 & NA & --- & --- & --- & --- \\
\hline $\begin{array}{l}\text { Araujo, 2004b } \\
\text { [55] }\end{array}$ & 3 & 3 & 2 & 2 & $\begin{array}{c}-- \\
\end{array}$ & --- & --- & --- \\
\hline Araujo, 2012 [56] & 3 & 3 & 3 & 2 & --- & --- & --- & --- \\
\hline $\begin{array}{l}\text { Araujo, 2012b } \\
\text { [57] }\end{array}$ & 3 & 3 & $2^{b}$ & 2 & --- & --- & --- & --- \\
\hline $\begin{array}{l}\text { Azevedo, } 2009 \\
\text { [58] }\end{array}$ & --- & --- & --- & --- & 2 & 2 & 2 & 3 \\
\hline Batista, 2012 [59] & 2 & 3 & 2 & 2 & 2 & 3 & 2 & NA \\
\hline $\begin{array}{l}\text { Calisher, } 1974 \\
{[60]}\end{array}$ & 3 & 3 & 2 & 3 & --- & --- & --- & --- \\
\hline Carrera, 2020 [61] & --- & --- & --- & --- & 3 & 3 & 3 & N/A \\
\hline Casseb, 2010 [62] & 3 & 3 & $2^{b}$ & 2 & --- & --- & --- & --- \\
\hline Casseb, 2016 [63] & 3 & 3 & $3^{b}$ & 2 & --- & --- & --- & --- \\
\hline $\begin{array}{l}\text { Catenacci, } 2017 \\
\text { [64] }\end{array}$ & 3 & 3 & 3 & N/A & 3 & 3 & 2 & 2 \\
\hline Cruz, 2009 [65] & 2 & 3 & 2 & N/A & --- & --- & --- & --- \\
\hline $\begin{array}{l}\text { Degallier, } 1992 \\
\text { [66] }\end{array}$ & 3 & 2 & 2 & 2 & 3 & 2 & 3 & N/A \\
\hline $\begin{array}{l}\text { De Thoisy, } 2003 \\
\text { [21] }\end{array}$ & 3 & 3 & 2 & 3 & --- & --- & --- & --- \\
\hline Diaz, 2007 [67] & 3 & 2 & 2 & 3 & --- & --- & --- & --- \\
\hline $\begin{array}{l}\text { Esposito, } 2015 \\
\text { [68] }\end{array}$ & --- & --- & --- & --- & $\begin{array}{l}\text { Unable to } \\
\text { judge }\end{array}$ & $\begin{array}{l}\text { Unable to } \\
\text { judge }\end{array}$ & $\begin{array}{l}\text { Unable to } \\
\text { judge }\end{array}$ & 3 \\
\hline Ferreira, 2020 [69] & --- & --- & --- & --- & 3 & 3 & 3 & 3 \\
\hline $\begin{array}{l}\text { Galindo, } 1966 \\
{[70]}\end{array}$ & 3 & 3 & 2 & N/A & 3 & 3 & 3 & 3 \\
\hline $\begin{array}{l}\text { Galindo, } 1967 \\
{[71]}\end{array}$ & --- & --- & --- & --- & 3 & 3 & 2 & 2 \\
\hline $\begin{array}{l}\text { Galindo, } 1983 \\
\text { [72] }\end{array}$ & 3 & 3 & 3 & N/A & 3 & 2 & 2 & 3 \\
\hline $\begin{array}{l}\text { GenBank } \\
\text { KY618129 }\end{array}$ & --- & --- & --- & --- & $\begin{array}{l}\text { Unable to } \\
\text { judge }\end{array}$ & $\begin{array}{l}\text { Unable to } \\
\text { judge }\end{array}$ & $\begin{array}{l}\text { Unable to } \\
\text { judge }\end{array}$ & 3 \\
\hline GenBank & --- & -- & --- & --- & Unable to & Unable to & Unable to & 3 \\
\hline
\end{tabular}




\begin{tabular}{|c|c|c|c|c|c|c|c|c|}
\hline KY618130 & & & & & judge & judge & judge & \\
\hline Gibrail, 2015 [73] & 3 & 3 & 2 & 2 & --- & --- & --- & --- \\
\hline Gomes, 2019 [74] & 3 & 3 & $3^{b}$ & 3 & --- & --- & --- & --- \\
\hline Groot, 1961 [75] & --- & --- & --- & --- & 3 & 3 & 3 & 3 \\
\hline Groot, 1964 [11] & 3 & 3 & 3 & 2 & --- & --- & --- & --- \\
\hline $\begin{array}{l}\text { Henriques, } 2008 \\
\text { [76] }\end{array}$ & --- & --- & --- & --- & 3 & 3 & 3 & N/A \\
\hline Hoch, 1981 [19] & 3 & 3 & 3 & 3 & 3 & 3 & 3 & 3 \\
\hline $\begin{array}{l}\text { Kubiszeski, } 2017 \\
\text { [77] }\end{array}$ & --- & --- & --- & --- & 3 & 3 & 3 & 2 \\
\hline $\begin{array}{l}\text { Laroque, } 2014 \\
{[78]}\end{array}$ & 3 & 3 & 2 & 2 & --- & --- & --- & --- \\
\hline Maia, 2019 [79] & --- & --- & --- & --- & 3 & 3 & 3 & 3 \\
\hline $\begin{array}{l}\text { Martinez, } 2020 \\
{[80]}\end{array}$ & --- & --- & --- & --- & 3 & 3 & 2 & N/A \\
\hline Medlin, 2016 [81] & 3 & 3 & 3 & N/A & --- & --- & --- & --- \\
\hline Medina, 2015 [82] & 3 & 2 & $2^{c}$ & 3 & --- & --- & --- & --- \\
\hline $\begin{array}{l}\text { Moreira-Soto, } \\
2018 \text { [83] }\end{array}$ & 3 & 3 & 3 & 3 & --- & --- & --- & --- \\
\hline Nunes, 2009 [84] & 2 & 3 & 2 & N/A & --- & --- & --- & --- \\
\hline Paulo, 2015 [50] & 3 & 3 & 3 & 2 & --- & --- & --- & --- \\
\hline $\begin{array}{l}\text { Pauvolid-Correa, } \\
2010 \text { [85] }\end{array}$ & 3 & 2 & $2^{b}$ & N/A & --- & --- & --- & --- \\
\hline $\begin{array}{l}\text { Pauvolid-Correa, } \\
2015 \text { [86] }\end{array}$ & 3 & 3 & 3 & 3 & --- & --- & --- & $\begin{array}{l}-- \\
\end{array}$ \\
\hline $\begin{array}{l}\text { Pauvolid-Correa, } \\
2008 \text { [87] }\end{array}$ & --- & --- & --- & --- & 3 & 3 & 2 & N/A \\
\hline Perez, 2019 [88] & 3 & 2 & 2 & 3 & --- & --- & --- & --- \\
\hline $\begin{array}{l}\text { Pinheiro, } 1974 \\
\text { [89] }\end{array}$ & 3 & 2 & 2 & 2 & 3 & 2 & 2 & N/A \\
\hline $\begin{array}{l}\text { Pinheiro, } 2019 \\
{[90]}\end{array}$ & --- & --- & --- & --- & 3 & 3 & 3 & N/A \\
\hline Powers, 2006 [91] & 3 & 2 & $\begin{array}{l}\text { Unable } \\
\text { to judge }\end{array}$ & 3 & 3 & 2 & $\begin{array}{l}\text { Unable to } \\
\text { judge }\end{array}$ & 3 \\
\hline Price, 1978 [92] & 3 & 2 & 2 & N/A & --- & --- & --- & --- \\
\hline Ragan, 2019 [93] & $\begin{array}{l}\text { Unable } \\
\text { to judge }\end{array}$ & $\begin{array}{l}\text { Unable } \\
\text { to judge }\end{array}$ & $\begin{array}{l}\text { Unable } \\
\text { to judge }\end{array}$ & $\begin{array}{l}\text { Unable to } \\
\text { judge }\end{array}$ & --- & --- & --- & --- \\
\hline $\begin{array}{l}\text { Sanmartin, } 1973 \\
{[94]}\end{array}$ & 3 & 3 & 3 & N/A & 2 & 3 & 2 & N/A \\
\hline Scherer, 1975 [95] & 2 & 3 & $3^{c}$ & N/A & 2 & 2 & 2 & N/A \\
\hline Serra, $2016[96]$ & --- & --- & --- & --- & 3 & 3 & 3 & 3 \\
\hline $\begin{array}{l}\text { Seymour, } 1983 \\
{[20]}\end{array}$ & 2 & 3 & 2 & 3 & --- & --- & --- & --- \\
\hline Silva, 2017 [97] & --- & --- & --- & --- & 3 & 3 & 3 & N/A \\
\hline $\begin{array}{l}\text { Srihongse, } 1974 \\
\text { [98] }\end{array}$ & 3 & 2 & 2 & 2 & --- & --- & --- & --- \\
\hline Tauro, 2019 [99] & --- & --- & --- & --- & 3 & 2 & 2 & N/A \\
\hline Taylor, 1967 [52] & $\begin{array}{l}\text { Unable } \\
\text { to judge }\end{array}$ & $\begin{array}{l}\text { Unable } \\
\text { to judge }\end{array}$ & $\begin{array}{l}\text { Unable } \\
\text { to judge }\end{array}$ & 3 & $\begin{array}{l}\text { Unable to } \\
\text { judge }\end{array}$ & $\begin{array}{l}\text { Unable to } \\
\text { judge }\end{array}$ & $\begin{array}{l}\text { Unable to } \\
\text { judge }\end{array}$ & 3 \\
\hline Turell, 2019 [100] & 3 & 2 & $3^{\mathrm{c}}$ & N/A & --- & --- & --- & --- \\
\hline
\end{tabular}

${ }^{a}$ Studies were assigned a score of NA for this criterion if no MAYV-positive samples were reported.

$327 \quad{ }^{\mathrm{b}}$ Domestic animals only.

$328{ }^{\mathrm{c}}$ Sentinel animals only. 


\section{MAYV in wild-caught non-human vertebrate animals}

Thirty-nine (68\%) studies in our systematic review assessed MAYV infection in wildinfection. Of the 27 taxonomic orders studied, 12 (44.4\%) had evidence of MAYV infection:

336 Artiodactyla (even-toed ungulates), Caprimulgiformes (nightbirds), Carnivora, Charadriiformes

337 (shorebirds), Cingulata (armadillos), Columbiformes (pigeons and doves), Didelphimorphia

338 (opossums), Passeriformes (passerine birds), Pilosa (sloths and anteaters), Primate, Rodentia, and

339 Squamata (scaled reptiles). The greatest number of MAYV-positive animal species were found

340 in the order Charadriiformes ( $n=16$ positive species) and the order Primate ( $n=15$ positive

341 species). (See S2 Table for complete mammal data and S3 Table for complete avian data).

Table 3 reports NHP species that were detected with MAYV antibodies. Only studies

343 with positive results are shown on Table 3; other negative studies are listed in the S2 Table.

344 High MAYV seroprevalence was confirmed by NT among Alouatta seniculus monkeys in

345 individual studies in French Guiana [21] $(\mathrm{n}=51 / 98)$ and among Callithrix argentata monkeys in

346 Brazil [19] (n=32/119). In addition, 29 Cebus libidinosus monkeys from wildlife screening

347 centers were detected with MAYV antibodies according to HI, although only six were reported

348 as monotypic reactions [78]. Diagnosis in these monkeys was not confirmed by NT. An

349 additional Cebus libidinosus monkey presented a heterotypic reaction to MAYV (titer of 1:20)

350 and four additional viruses according to HI (including a titer of 1:640 for Oropouche virus) [73]. 
351 However, based on the study's protocol, confirmatory NT was only performed for viruses with

352 titers $\geq 1: 40$.

353 Table 3. Evidence of MAYV infection in non-human primates

\begin{tabular}{|c|c|c|c|c|c|c|}
\hline Species & $\begin{array}{c}\text { Positive } \\
\text { (n) }\end{array}$ & $\begin{array}{c}\text { Total } \\
\text { tested }(\mathbf{n})^{a}\end{array}$ & $\%$ Pos & Test method & Notes & Citation \\
\hline \multirow[t]{2}{*}{ Alouatta seniculus } & 51 & 98 & 52.0 & $\begin{array}{l}\text { HI with } \\
\text { confirmatory } \\
\text { NT }^{\mathrm{d}}\end{array}$ & NA & [21] \\
\hline & 1 & 1 & 100.0 & $\begin{array}{l}\text { ELISA with } \\
\text { confirmatory } \\
\text { plaque-reduction } \\
\text { NT }\end{array}$ & NA & [88] \\
\hline $\begin{array}{l}\text { Callithrix } \\
\text { argentata }\end{array}$ & 32 & 119 & 26.9 & $\begin{array}{l}\text { HI with } \\
\text { confirmatory NT }\end{array}$ & $\begin{array}{l}\text { One isolation also reported but not } \\
\text { included in this table. }\end{array}$ & [19] \\
\hline Cebus libidinosus $^{\mathrm{b}}$ & 6 & 100 & 6.0 & $\mathrm{HI}$ & $\begin{array}{l}\text { Six reactions were monotypic, and } \\
23 \text { were heterotypic, with titers of } \\
1: 20(n=1), 1: 80(n=6), 1: 160 \\
(n=2), 1: 320(n=6), 1: 640(n=6), \\
\text { and } 1: 1280(n=8) \text {. Only } 6 \text { of the } 29 \\
\text { reactions were monotypic. }\end{array}$ & [78] \\
\hline $\begin{array}{l}\text { Tamarin, Pithecia, } \\
\text { Cebus (species not } \\
\text { specified) }\end{array}$ & 7 & 21 & 33.3 & $\mathrm{HI}$ & $\begin{array}{l}\text { Results presented as a table from } \\
\text { the Belem Virus Laboratory, but } \\
\text { no further information is provided } \\
\text { regarding the study methods or } \\
\text { primate species. }\end{array}$ & [52] \\
\hline Cebus apella & 10 & 62 & 16.1 & $\mathrm{HI}$ & $\begin{array}{l}\text { Titer results for monotypic } \\
\text { reactions were } 1: 80(\mathrm{n}=2), 1: 160 \\
(\mathrm{n}=7) \text { and } 1: 640(\mathrm{n}=1) \text {. Three } \\
\text { additional samples showed positive } \\
\text { results for MAYV and another } \\
\text { virus. }\end{array}$ & [59] \\
\hline Saguinas midas & 8 & 42 & 19.1 & $\begin{array}{l}\text { HI with } \\
\text { confirmatory } \\
\text { NT }^{\mathrm{d}}\end{array}$ & NA & [21] \\
\hline Alouatta sp. ${ }^{\mathrm{c}}$ & 7 & 11 & 63.6 & $\mathrm{HI}$ & NA & [11] \\
\hline $\begin{array}{l}\text { Lagothrix } \\
\text { poeppigii }\end{array}$ & 6 & 11 & 54.5 & $\begin{array}{l}\text { ELISA with } \\
\text { confirmatory } \\
\text { plaque-reduction } \\
\text { NT }\end{array}$ & NA & [88] \\
\hline Saimiri sciureus & 4 & 6 & 66.7 & $\begin{array}{l}\text { HI with } \\
\text { confirmatory } \\
\text { NT }^{\mathrm{d}}\end{array}$ & NA & [21] \\
\hline Pithecia pithecia & 4 & 5 & 80.0 & $\begin{array}{l}\text { HI with } \\
\text { confirmatory } \\
\text { NT }^{\mathrm{d}}\end{array}$ & NA & [21] \\
\hline Cebus sp. ${ }^{\mathrm{C}}$ & 4 & 13 & 30.8 & HI & NA & [11] \\
\hline Alouatta villosa & 3 & 5 & 60.0 & $\begin{array}{l}\text { Plaque-reduction } \\
\text { NT }\end{array}$ & $\begin{array}{l}\text { Samples considered positive if } \\
90 \% \text { plaque reduction by plasma } \\
1: 16 \text { or weaker. The median } \\
\text { positive titer was } 1: 128 \text { (range }\end{array}$ & [20] \\
\hline
\end{tabular}




\begin{tabular}{|c|c|c|c|c|c|c|}
\hline & & & & & 1:32-1:512). & \\
\hline Sapajus sp. & 3 & 43 & 7.0 & HI and RT-PCR & $\begin{array}{l}\text { Positive samples had a monotypic } \\
\text { reaction to MAYV with titers of } \\
1: 80(n=1) \text { and } 1: 160(n=2) \text {. All } \\
\text { samples negative by RT-PCR. }\end{array}$ & {$[50]$} \\
\hline $\begin{array}{l}\text { Sapajus } \\
\text { xanthosternos }\end{array}$ & 1 & 2 & $\mathbf{5 0 . 0}$ & $\begin{array}{l}\text { Plaque-reduction } \\
\text { NT }\end{array}$ & $\begin{array}{l}\text { Plaque reduction NTs were } \\
\text { performed against MAYV for all } \\
\text { CHIKV-positive samples. The } \\
\text { sample neutralized both MAYV } \\
\text { and CHIKV at titers of 1:40. }\end{array}$ & [83] \\
\hline Ateles marginatus & 1 & 1 & 100.0 & $\begin{array}{l}\text { Plaque-reduction } \\
\text { NT }\end{array}$ & $\begin{array}{l}\text { Plaque reduction NTs were } \\
\text { performed against MAYV for all } \\
\text { CHIKV-positive samples. The } \\
\text { sample neutralized both MAYV } \\
\text { and CHIKV at titers of 1:40. }\end{array}$ & [83] \\
\hline Alouatta belzebul & 1 & 1 & 100.0 & $\begin{array}{l}\text { HI with } \\
\text { confirmatory NT }\end{array}$ & NA & {$[19]$} \\
\hline $\begin{array}{l}\text { Sapajus } \\
\text { macrocephalus }\end{array}$ & 1 & 6 & 16.7 & $\begin{array}{l}\text { ELISA with } \\
\text { confirmatory } \\
\text { plaque-reduction } \\
\text { NT }\end{array}$ & NA & {$[88]$} \\
\hline Cacajao calvus & 1 & 3 & 33.3 & $\begin{array}{l}\text { ELISA with } \\
\text { confirmatory } \\
\text { plaque-reduction } \\
\text { NT }\end{array}$ & NA & [88] \\
\hline $\begin{array}{l}\text { Callicebus } \\
\text { brunneus }^{\mathrm{e}}\end{array}$ & 1 & N/A & NA & $\mathrm{HI}$ & $\begin{array}{l}\text { Sera reacted against MAYV and } \\
\text { Tacaiuma virus. No additional } \\
\text { information provided. }\end{array}$ & {$[66]$} \\
\hline Aotus sp. ${ }^{\mathrm{c}}$ & 1 & 4 & 25.0 & $\mathrm{HI}$ & NA & [11] \\
\hline Saimiri sp. ${ }^{\mathrm{c}}$ & 1 & 1 & 100.0 & $\mathrm{HI}$ & NA & [11] \\
\hline
\end{tabular}

MAYV: Mayaro virus; HI: hemagglutination inhibition; ELISA: enzyme-linked immunosorbent assay; RT-PCR: reverse transcription polymerase chain reaction; NT: neutralization test; CHIKV: Chikungunya virus

${ }^{a}$ Denominators presented in this table reflect only studies that reported MAYV positivity. Complete data (including MAYV-negative samples) are included in the seroprevalence meta-analysis and the Supplementary Tables.

${ }^{\mathrm{b}}$ Captive primates from a wildlife rescue facility.

${ }^{\mathrm{c}}$ Sera analyzed for MAYV may have had cross reactivity with Una virus because the authors used a Colombian isolate that was initially characterized as MAYV but was later identified as Una virus. A differential test was not performed for MAYV. However, the authors identified human sera that was reactive to MAYV alone in the same study region.

${ }^{\mathrm{d}}$ Serum samples with titers $>1: 20$ confirmed by seroneutralization. Positive reaction was considered with the total inhibition of the cytopathic effect in the cell monolayer.

${ }^{\mathrm{e}}$ Authors also reported that seven monkey sera among the 14 examined were positive for yellow fever and MAYV, of which five were positive for the two agents. The species of these positive samples were: Pithecia pithecia $(\mathrm{n}=1)$, Alouatta seniculus $(\mathrm{n}=2)$, Saimiri sciureus $(\mathrm{n}=1)$, Saguinus midas $(\mathrm{n}=1)$, and Ateles paniscus $(\mathrm{n}=2)$. However, they did not note the specific primate species that were positive for MAYV.

371 confirmed by NT and three by HI alone. In addition, MAYV positivity was reported in the

372 following NHP genera, although animals were not reported to species: Aotus ( $\mathrm{n}=1 / 4)$, Alouatta 
$373(\mathrm{n}=7 / 11)$, Cebus $(\mathrm{n}=4 / 13)$, Sapajus $(\mathrm{n}=3 / 43)$, and Saimiri $(\mathrm{n}=1 / 1)$. The authors reporting MAYV

374 positivity in the Aotus, Alouatta, Cebus, and Saimiri genera noted that these results should be

375 interpreted with caution due to potential for cross-reactivity with Una virus (UNAV) [11]. In one

376 study conducted in Brazil, two of 11 Chikungunya virus (CHIKV)-positive serum samples (in

377 the species Sapajus xanthosternos and Ateles marginatus) neutralized MAYV with titers of 1:40

378 in plaque reduction NTs [83]. These two samples were considered MAYV-positive and included

379 in our meta-analysis. One additional study [67] detected neutralizing antibodies against both

380 UNAV and MAYV in 21 Alouatta caraya monkeys. However, all 21 monkeys were diagnosed

381 with UNAV based on a 4-fold titer difference between the two viruses. Therefore, we considered

382 these monkeys MAYV-negative and did not include them in our meta-analysis. Finally, in 1963

383 the Belem Virus laboratory reported MAYV infection in seven NHPs based on HI tests alone

384 [52]. These monkeys were described as Tamarin, Pithecia, and Cebus although no further

385 information was provided regarding sampling method, testing protocol, or primate species.

386 MAYV antibodies were also detected in 21 bird species from the order Charadriiformes

$387(n=16)$ and Passeriformes $(n=5)$. All MAYV-positive birds were found in Brazil, with the

388 exception of one MAYV isolate from a migrating bird captured in Louisiana USA [60]. A high

389 MAYV-seroprevalence $(n=34 / 122)$ was reported by the Belem Laboratory in 1963 among

390 Columbigallina birds, although no additional information was provided regarding sampling

391 method or bird species. MAYV antibodies were also detected in seven avian families that were

392 not identified to genus or species. Only one study that detected MAYV antibodies in birds

393 performed confirmatory NT [19]. All other diagnoses (with the exception of the virus isolation)

394 were made by HI tests alone. See Table 4 for additional information regarding avian species that 395 were infected with MAYV. 
Table 4. Evidence of MAYV infection in birds

\begin{tabular}{|c|c|c|c|c|c|c|c|}
\hline Order & Species & $\begin{array}{c}\text { Positive } \\
\text { (n) }\end{array}$ & $\begin{array}{c}\text { Total } \\
(\mathbf{n})^{\mathrm{a}}\end{array}$ & $\%$ Pos & Test method & Notes & Citation \\
\hline Columbiformes & Columbigallina sp. & 34 & 121 & 28.1 & $\mathrm{HI}$ & $\begin{array}{l}\text { Results presented as a } \\
\text { table from the Belem } \\
\text { Virus Laboratory, but } \\
\text { no further information } \\
\text { is provided regarding } \\
\text { the methods or species. }\end{array}$ & [52] \\
\hline Charadriiformes & Sterna hirundo & 23 & 342 & 6.7 & $\mathrm{HI}$ & NA & [53] \\
\hline Charadriiformes & Sterna trudeaui & 12 & 56 & 21.4 & $\mathrm{HI}$ & NA & [53] \\
\hline \multirow[t]{2}{*}{ Charadriiformes } & \multirow[t]{2}{*}{ Arenaria interpres } & 8 & 28 & 28.6 & $\mathrm{HI}$ & NA & [53] \\
\hline & & 1 & NA & NA & HI & Titers 1:40 & [55] \\
\hline Charadriiformes & Calidris canutus & 7 & 51 & 13.7 & $\mathrm{HI}$ & NA & [53] \\
\hline Passeriformes & $\begin{array}{l}\text { Fringillidae family, } \\
\text { unspecified species }\end{array}$ & 6 & 131 & 4.6 & $\begin{array}{l}\text { HI with } \\
\text { confirmatory } \\
\text { NT }\end{array}$ & NA & [19] \\
\hline Passeriformes & $\begin{array}{l}\text { Formicariidae } \\
\text { family, unspecified } \\
\text { species }\end{array}$ & 5 & 444 & 1.1 & $\begin{array}{l}\text { HI with } \\
\text { confirmatory } \\
\text { NT }\end{array}$ & NA & [19] \\
\hline Charadriiformes & Limosa haemastica & 5 & 17 & 29.4 & $\mathrm{HI}$ & NA & [53] \\
\hline Charadriiformes & Tringa flavipes & 4 & 5 & 80.0 & HI & NA & [53] \\
\hline \multirow[t]{2}{*}{ Charadriiformes } & \multirow[t]{2}{*}{ Calidris pusilla } & 3 & NA & NA & $\mathrm{HI}$ & $\begin{array}{l}\text { Titers 1:40 for all } \\
\text { positive samples }\end{array}$ & [55] \\
\hline & & 1 & 30 & 3.3 & $\mathrm{HI}$ & $\begin{array}{l}\text { Monotypic reaction } \\
\text { with titers } \geq 1: 20 \text { to } \\
\text { MAYV }\end{array}$ & {$[56]$} \\
\hline Charadriiformes & $\begin{array}{l}\text { Sterna } \\
\text { superciliaris }\end{array}$ & 2 & 8 & 25.0 & $\mathrm{HI}$ & N/A & {$[53]$} \\
\hline Charadriiformes & Actitis macularius & 2 & 22 & 9.1 & $\mathrm{HI}$ & $\begin{array}{l}\text { Monotypic reaction } \\
\text { with titers } \geq 1: 20 \text { to } \\
\text { MAYV }\end{array}$ & {$[56]$} \\
\hline Passeriformes & $\begin{array}{l}\text { Dendrocolaptidae } \\
\text { family, unspecified } \\
\text { species }\end{array}$ & 1 & 97 & 1.0 & $\begin{array}{l}\text { HI with } \\
\text { confirmatory } \\
\text { NT }\end{array}$ & NA & [19] \\
\hline Passeriformes & Icterus spurius & 1 & 223 & 0.45 & $\begin{array}{l}\text { Virus } \\
\text { isolation by } \\
\text { inoculation } \\
\text { into suckling } \\
\text { mice }\end{array}$ & NA & {$[60]$} \\
\hline Passeriformes & $\begin{array}{l}\text { Arremon } \\
\text { tactiturnus }\end{array}$ & 1 & NA & NA & $\begin{array}{l}\text { HI } \\
\text { (confirmatory } \\
\text { NT unclear) }\end{array}$ & NA & {$[66]$} \\
\hline Passeriformes & $\begin{array}{l}\text { Pipridae family, } \\
\text { unspecified species }\end{array}$ & 1 & 229 & 0.44 & $\begin{array}{l}\text { HI with } \\
\text { confirmatory } \\
\text { NT }\end{array}$ & NA & [19] \\
\hline Passeriformes & $\begin{array}{l}\text { Cercomacra } \\
\text { tyrannina }\end{array}$ & 1 & NA & NA & $\begin{array}{l}\text { HI } \\
\text { (confirmatory } \\
\text { NT unclear) }\end{array}$ & NA & {$[66]$} \\
\hline Passeriformes & $\begin{array}{l}\text { Formicivora } \\
\text { grisea }\end{array}$ & 1 & NA & NA & $\begin{array}{l}\text { HI } \\
\text { (confirmatory } \\
\text { NT unclear) }\end{array}$ & NA & [66] \\
\hline Passeriformes & Tyrannus & 1 & NA & NA & HI & NA & [66] \\
\hline
\end{tabular}




\begin{tabular}{|c|c|c|c|c|c|c|c|}
\hline & melancholicus & & & & $\begin{array}{l}\text { (confirmatory } \\
\text { NT unclear) }\end{array}$ & & \\
\hline Passeriformes & $\begin{array}{l}\text { Tyrannidae family, } \\
\text { unspecified species }\end{array}$ & 1 & 102 & 0.98 & $\begin{array}{l}\text { HI with } \\
\text { confirmatory } \\
\text { NT }\end{array}$ & NA & [19] \\
\hline Charadriiformes & $\begin{array}{l}\text { Pluvialis } \\
\text { squatarola }\end{array}$ & 1 & 4 & 25.0 & $\mathrm{HI}$ & $\begin{array}{l}\text { Monotypic reaction } \\
\text { with titers } \geq 1: 20 \text { to } \\
\text { MAYV }\end{array}$ & [56] \\
\hline Charadriiformes & $\begin{array}{l}\text { Haematopus } \\
\text { palliatus }\end{array}$ & 1 & 6 & 16.7 & $\mathrm{HI}$ & NA & [53] \\
\hline Charadriiformes & Sterna eurygnatha & 1 & 7 & 14.3 & $\mathrm{HI}$ & NA & [53] \\
\hline Charadriiformes & Sterna maxima & 1 & 1 & 100 & $\mathrm{HI}$ & NA & [53] \\
\hline Charadriiformes & Sterna niotica & 1 & 1 & 100 & $\mathrm{HI}$ & NA & [53] \\
\hline Charadriiformes & Calidris fuscicollis & 1 & 11 & 9.1 & HI & NA & [53] \\
\hline Charadriiformes & Calidris minutilla & 1 & 6 & 16.7 & $\mathrm{HI}$ & $\begin{array}{l}\text { Monotypic reaction } \\
\text { with titers } \geq 1: 20 \text { to } \\
\text { MAYV }\end{array}$ & [56] \\
\hline Caprimulgiformes & $\begin{array}{l}\text { Caprimulgidae } \\
\text { family, unspecified } \\
\text { species }\end{array}$ & 1 & 5 & 20.0 & $\begin{array}{l}\text { HI with } \\
\text { confirmatory } \\
\text { NT }\end{array}$ & NA & [19] \\
\hline Columbiformes & $\begin{array}{l}\text { Columbidae } \\
\text { family, unspecified } \\
\text { species }\end{array}$ & 1 & 34 & 2.9 & $\begin{array}{l}\text { HI with } \\
\text { confirmatory } \\
\text { NT }\end{array}$ & NA & [19] \\
\hline Passeriformes & Molothrus sp. & 1 & NA & NA & HI & Titers 1:80 & [55] \\
\hline
\end{tabular}

MAYV: Mayaro virus; HI: hemagglutination inhibition; NT: neutralization test

${ }^{a}$ Denominators presented in this table reflect only studies that reported MAYV positivity. Complete data (including

MAYV-negative samples) is reflected in the seroprevalence meta-analysis and the Supplementary Tables.

402 Table 5. Six rodent species as well as unidentified rodents in the Echimys and Proechimys

403 genera were detected with MAYV antibodies in French Guiana [21], Peru [88], and Panama

404 [20]. In addition, four species in the order Didelphimorphia, three species in the order Pilosa, and

405 one species each in the orders Carnivora, Artiodactyla, and Cingulata were detected with MAYV

406 antibodies in French Guiana [21] and Peru [88]. Additional positive samples were detected in the

407 orders Rodentia, Didelphimorphia, and Pilosa although the species were not identified.

408 Table 5. Evidence of MAYV infection in mammals (excluding non-human primates)

\begin{tabular}{|l|l|c|c|c|l|l|l|}
\hline Order & Species & $\begin{array}{c}\text { Positive } \\
(\mathbf{n})\end{array}$ & $\begin{array}{c}\text { Total } \\
(\mathbf{n})^{\mathbf{a}}\end{array}$ & \% Pos & Test method & Notes & Citation \\
\hline Rodentia & $\begin{array}{l}\text { Wild rodents, } \\
\text { unspecified }\end{array}$ & 71 & 960 & $\mathbf{7 . 4}$ & HI & $\begin{array}{l}\text { Results presented as a table } \\
\text { from the Belem Virus } \\
\text { Laboratory, but no further }\end{array}$ & {$[52]$} \\
\hline
\end{tabular}




\begin{tabular}{|c|c|c|c|c|c|c|c|}
\hline & & & & & & $\begin{array}{l}\text { information is provided } \\
\text { regarding the methods or } \\
\text { species. }\end{array}$ & \\
\hline Didelphimorphia & $\begin{array}{l}\text { Opossum, } \\
\text { unspecified }\end{array}$ & 9 & 122 & 7.4 & $\mathrm{HI}$ & $\begin{array}{l}\text { Results presented as a table } \\
\text { from the Belem Virus } \\
\text { Laboratory, but no further } \\
\text { information is provided } \\
\text { regarding the methods or } \\
\text { species. }\end{array}$ & {$[52]$} \\
\hline Pilosa & $\begin{array}{l}\text { Choloepus } \\
\text { didactylus }\end{array}$ & 7 & 26 & 26.9 & $\begin{array}{l}\text { HI with } \\
\text { confirmatory } \\
\text { NT }^{\mathrm{b}}\end{array}$ & NA & [21] \\
\hline Didelphimorphia & Marmosa sp. & 7 & 46 & 15.2 & $\mathrm{HI}$ & NA & [52] \\
\hline Pilosa & $\begin{array}{l}\text { Tamandua } \\
\text { tetradactyla }\end{array}$ & 6 & 26 & 23.1 & $\begin{array}{l}\text { HI with } \\
\text { confirmatory } \\
\mathrm{NT}^{\mathrm{b}}\end{array}$ & NA & [21] \\
\hline \multirow[t]{2}{*}{ Cingulata } & \multirow[t]{2}{*}{$\begin{array}{l}\text { Dasypus } \\
\text { novemcinctus }\end{array}$} & 4 & 40 & 10.0 & $\begin{array}{l}\text { HI with } \\
\text { confirmatory } \\
\text { NT }^{\mathrm{b}}\end{array}$ & NA & [21] \\
\hline & & 2 & 4 & 50.0 & $\begin{array}{l}\text { ELISA with } \\
\text { confirmatory } \\
\text { plaque } \\
\text { reduction NT }\end{array}$ & NA & {$[88]$} \\
\hline Rodentia & $\begin{array}{l}\text { Dasyprocta } \\
\text { leporina }\end{array}$ & 5 & 29 & 17.2 & $\begin{array}{l}\text { HI with } \\
\text { confirmatory } \\
\mathrm{NT}^{\mathrm{b}}\end{array}$ & NA & [21] \\
\hline Didelphimorphia & $\begin{array}{l}\text { Philander } \\
\text { opossum }\end{array}$ & 5 & 27 & 18.5 & $\begin{array}{l}\text { HI with } \\
\text { confirmatory } \\
\text { NT }^{\mathrm{b}}\end{array}$ & NA & [21] \\
\hline Rodentia & $\begin{array}{l}\text { Coendou } \\
\text { prehensilis }\end{array}$ & 3 & 26 & 11.5 & $\begin{array}{l}\text { HI with } \\
\text { confirmatory } \\
\text { NT }^{\mathrm{b}}\end{array}$ & NA & [21] \\
\hline Rodentia & $\begin{array}{l}\text { Dasyprocta } \\
\text { punctata }\end{array}$ & 3 & 5 & 60.0 & $\begin{array}{l}\text { Plaque } \\
\text { reduction NT }\end{array}$ & $\begin{array}{l}\text { Samples considered } \\
\text { positive if } 90 \% \text { plaque } \\
\text { reduction by plasma } 1: 16 \text { or } \\
\text { weaker. The median } \\
\text { positive titer was } 1: 128 \\
\text { (range } 1: 32-1: 512 \text { ). }\end{array}$ & [20] \\
\hline Rodentia & $\begin{array}{l}\text { Dasyprocta } \\
\text { fuliginosa }\end{array}$ & 3 & 27 & 11.1 & $\begin{array}{l}\text { ELISA with } \\
\text { confirmatory } \\
\text { plaque } \\
\text { reduction NT }\end{array}$ & NA & [88] \\
\hline Rodentia & $\begin{array}{l}\text { Coendou } \\
\text { melanurus }\end{array}$ & 2 & 15 & $\mathbf{1 3 . 3}$ & $\begin{array}{l}\text { HI with } \\
\text { confirmatory } \\
\text { NT }^{\mathrm{b}}\end{array}$ & NA & {$[21]$} \\
\hline Didelphimorphia & $\begin{array}{l}\text { Didelphis } \\
\text { albiventris }\end{array}$ & 2 & 19 & 10.5 & $\begin{array}{l}\text { HI with } \\
\text { confirmatory } \\
\text { NT }^{\mathrm{b}}\end{array}$ & NA & [21] \\
\hline Rodentia & Echimys sp. & 1 & 21 & 4.8 & $\begin{array}{l}\text { HI with } \\
\text { confirmatory } \\
\text { NT }^{\mathrm{b}}\end{array}$ & NA & [21] \\
\hline Rodentia & Agouti paca & 1 & 10 & 10.0 & $\begin{array}{l}\text { ELISA with } \\
\text { confirmatory } \\
\text { plaque } \\
\text { reduction NT }\end{array}$ & NA & [88] \\
\hline
\end{tabular}




\begin{tabular}{|l|l|c|c|c|l|l|l|}
\hline Rodentia & $\begin{array}{l}\text { Proechimys } \\
\text { sp. }\end{array}$ & 1 & 18 & $\mathbf{5 . 6}$ & $\begin{array}{l}\text { HI with } \\
\text { confirmatory } \\
\text { NT }\end{array}$ & NA & {$[21]$} \\
\hline Didelphimorphia & $\begin{array}{l}\text { Caluromys } \\
\text { philander }\end{array}$ & 1 & 5 & $\mathbf{2 0 . 0}$ & $\begin{array}{l}\text { HI with } \\
\text { confirmatory } \\
\text { NT }\end{array}$ & NA & [21] \\
\hline Didelphimorphia & $\begin{array}{l}\text { Didelphis } \\
\text { marsupialis }\end{array}$ & 1 & 29 & $\mathbf{3 . 5}$ & $\begin{array}{l}\text { HI with } \\
\text { confirmatory } \\
\text { NT }\end{array}$ & NA & {$[21]$} \\
\hline Carnivora & Potos flavus & 1 & 9 & $\mathbf{1 1 . 1}$ & $\begin{array}{l}\text { HI with } \\
\text { confirmatory } \\
\text { NT }\end{array}$ & NA & {$[21]$} \\
\hline Artiodactyla & Pecari tajacu & 1 & 6 & $\mathbf{1 6 . 7}$ & $\begin{array}{l}\text { ELISA with } \\
\text { confirmatory } \\
\text { plaque } \\
\text { reduction NT }\end{array}$ & NA & {$[88]$} \\
\hline Pilosa & $\begin{array}{l}\text { Bradypus } \\
\text { tridactylus }\end{array}$ & 1 & 29 & $\mathbf{3 . 5}$ & $\begin{array}{l}\text { HI with } \\
\text { confirmatory } \\
\text { NT }\end{array}$ & NA & {$[21]$} \\
\hline Pilosa & Bradypus sp. & 1 & 3 & $\mathbf{3 3 . 3}$ & HI & NA & {$[52]$} \\
\hline
\end{tabular}

MAYV: Mayaro virus; HI: hemagglutination inhibition; ELISA: enzyme-linked immunosorbent assay; RT-PCR: reverse transcription polymerase chain reaction; NT: neutralization test

${ }^{a}$ Denominators presented in this table reflect only studies that reported MAYV positivity. Complete data (including

414 inhibition of the cytopathic effect in the cell monolayer.

417 silvery marmoset (Callithrix argentata) captured during a MAYV outbreak in Belterra, Brazil

418 [19] and a migrating orchard oriole (Icterus spurius) captured in Louisiana [60]. In addition, the

419 Belem Virus Laboratory reported MAYV isolation from two lizard species in 1963 [52]

420 (Tropidurus torquatus and Ameiva ameiva) although no further information was provided

421 regarding study methods or procedures.

423 with MAYV is presented in Fig 2. The infected animals were identified in six countries overall,

424 including Brazil, Peru, French Guiana, Colombia, Venezuela, and Panama, although the majority

425 of infected animals were found in Brazil. Overall, 12 locations were geo-referenced as points, 
four locations as ADM1 polygons, 15 locations as ADM2 polygons, and two locations as custom

427 polygons.

Fig 2. Georeferenced locations of MAYV positivity in non-human animals and arthropods. The finest spatial scale is presented where possible. One MAYV isolate detected in a migrating bird in Louisiana is not included in the map.

\section{MAYV in domestic or sentinel animals}

Nine studies analyzed MAYV seroprevalence in domestic animals (equids, sheep,

434 poultry, dogs, pigs, cattle, and buffaloes), and five studies analyzed MAYV seroprevalence in

435 sentinel animals (monkeys, mice, and hamsters). Domestic and sentinel animals with evidence

436 of MAYV positivity are reported in Table $\mathbf{5}$ and complete results are reported in the S4 Table.

437 In domestic animals, evidence of MAYV infection was detected in equids, cattle/buffalo, and

438 dogs. Six studies assessed MAYV seroprevalence in Brazilian equids [54, 57, 63, 74, 85, 86],

439 and antibodies against MAYV were detected in four of these studies. Notably, Gomes et al. [74]

440 reported MAYV neutralizing antibodies in 48 equids out of 213 (23\%) based on ELISA.

441 However, only 16 of the 48 equids were considered positive based on the study's diagnostic

442 criterion of 4-fold greater plaque reduction $\mathrm{NT}_{90}$ titer than that of the other viruses under study.

443 In addition, Casseb et al. [63] detected MAYV antibodies in 40 horses using HI, although only

444 four of the 40 reactions were monotypic, and confirmatory NTs were not performed. Additional

445 domestic animals with evidence of MAYV infection included cattle/buffalo ( $n=14 / 1103$ positive

446 reactions by $\mathrm{HI}$; 5/14 monotypic reactions [62]) and dogs ( $\mathrm{n}=2 / 7$ positive reactions by HI [53]).

447 In addition, neutralizing antibodies (plaque reduction $\mathrm{NT}_{90}$ titer $\geq 10$ ) against MAYV were

448 detected in three sheep in Brazil [86]. However, these animals did not meet the original study's

449 diagnostic criterion for MAYV diagnosis based on 4-fold greater plaque reduction $\mathrm{NT}_{90}$ titer 
451 in two sentinel monkeys placed in the tree canopy in Panama [98], and one MAYV isolate was

452 obtained from a sentinel hamster in Venezuela [82].

453 Table 6. Domestic and sentinel animals with evidence of MAYV infection

\begin{tabular}{|c|c|c|c|c|c|c|}
\hline Animal Type & $\begin{array}{c}\text { Total } \\
\text { Positive } \\
\end{array}$ & $\begin{array}{c}\text { Number } \\
\text { Tested }^{\text {a }}\end{array}$ & $\%$ Pos & Test Method & Notes & Citation \\
\hline \multirow[t]{4}{*}{$\begin{array}{l}\text { Domestic } \\
\text { Equids }\end{array}$} & 16 & 213 & $\overline{7.5}$ & $\begin{array}{l}\text { ELISA with } \\
\text { confirmatory } \\
\text { plaque reduction } \\
\text { NT }\end{array}$ & $\begin{array}{l}\text { Forty-eight horses had } \\
\text { antibodies to MAYV by } \\
\text { ELISA. Sixteen of } 48(33 \%) \\
\text { were considered positive by } \\
\left.{\text { plaque reduction } \mathrm{NT}_{90} \text { for }} \text { MAYV with titers } 1: 10(\mathrm{n}=12),_{1} \text {, } 1: 20=3\right) \text { and } 1: 40(\mathrm{n}=1) .\end{array}$ & {$[74]$} \\
\hline & 4 & 753 & 0.5 & $\mathrm{HI}$ & $\begin{array}{l}\text { Forty reactions overall. Four of } \\
40 \text { reactions were monotypic } \\
\text { while } 36 \text { of } 40 \text { were } \\
\text { heterotypic. }\end{array}$ & [63] \\
\hline & 11 & 102 & 10.8 & $\mathrm{HI}$ & $\begin{array}{l}\text { Not clear if the } 11 \text { reactions are } \\
\text { monotypic or heterotypic. }\end{array}$ & {$[57]$} \\
\hline & 10 & 748 & 1.5 & $\begin{array}{l}\text { Plaque reduction } \\
\text { NT }\end{array}$ & $\begin{array}{l}\text { Forty-four horses had } \\
\text { neutralizing antibody (titer } \geq \\
\text { 10) against MAYV, but only } \\
\text { ten met the diagnostic criteria of } \\
\text { 4-fold greater plaque reduction } \\
\mathrm{NT}_{90} \text { titer than the three other } \\
\text { viruses (VEEV, EEEV, } \\
\text { WEEV). Positive samples had } \\
\text { titers of } 1: 20(\mathrm{n}=6) \text { and } 1: 40 \\
(\mathrm{n}=4)\end{array}$ & {$[86]$} \\
\hline $\begin{array}{l}\text { Domestic } \\
\text { Cattle/Buffalo }\end{array}$ & 5 & 1103 & 0.5 & $\mathrm{HI}$ & $\begin{array}{l}\text { Positive reactions were } \\
\text { considered any reaction with a } \\
\text { titer equal to or greater than } \\
1: 20 \text {. Fourteen reactions overall, } \\
\text { and five of } 14 \text { reactions were } \\
\text { monotypic. }\end{array}$ & {$[62]$} \\
\hline $\begin{array}{l}\text { Domestic } \\
\text { Dog }\end{array}$ & 2 & 7 & 28.6 & $\mathrm{HI}$ & N/A & {$[53]$} \\
\hline $\begin{array}{l}\text { Sentinel } \\
\text { Hamster }\end{array}$ & 1 & N/A & N/A & RT-PCR & & [82] \\
\hline $\begin{array}{l}\text { Sentinel } \\
\text { Monkeys }\end{array}$ & 2 & 13 & 15.4 & HI & N/A & [98] \\
\hline
\end{tabular}

MAYV: Mayaro virus; HI: hemagglutination inhibition; ELISA: enzyme-linked immunosorbent assay; RT-PCR: reverse transcription polymerase chain reaction; NT: neutralization test; VEEV: Venezuelan equine encephalitis virus; EEEV: Eastern equine encephalitis virus; WEEV: Western equine encephalitis virus

$457{ }^{a}$ Denominators presented in this table reflect only studies that reported MAYV positivity. Complete data (including 


\section{Pooled prevalence of MAYV in non-human vertebrate animals}

Twenty-four studies overall were included in the pooled prevalence meta-analysis. Eight

462 studies were excluded because they did not clearly state how many animals were tested for

463 MAYV within each order [55, 66, 72, 84, 89, 93] or did not present serologic results [60, 70].

464 Another study was excluded because authors reported the number of "Group A" positive serum

465 samples, but did not specify individual viruses [98]. Studies were also excluded if they only

466 reported sequence data or only included sentinel animals $[82,91,95,100]$. Finally, a study that

467 sampled bats exclusively was excluded because no MAYV-positive samples were reported in the

468 order Chiroptera [92].

Eleven orders of nonhuman vertebrate animals (including domestic equids) were included

470 in the meta-analysis. Orders were excluded from the analysis due to insufficient sample size

$471(\mathrm{~N}<10)$ or if no MAYV-positive samples were reported. These include the orders Apodiformes

472 (MAYV prevalence: 0/3), Caprimulgiformes (MAYV prevalence: 1/6), Chiroptera (MAYV

473 prevalence: 0/1546), Crocodilia (MAYV prevalence: 0/87), Cuculiformes (MAYV prevalence:

474 0/5), Galliformes (MAYV prevalence: 0/1), Gruiformes (MAYV prevalence: 0/2), Psittaciformes

475 (MAYV prevalence: 0/3), Tinamiformes (MAYV prevalence: 0/2), Pelecaniformes (MAYV

476 prevalence: 0/2), and Podicipediformes (MAYV prevalence: 0/2).

The primate order appeared in 14 studies that were included in the meta-analysis. When

478 all positive samples were included, the pooled MAYV seroprevalence among primates was

$47913.1 \%$ (95\% CI: 4.3-25.1\%) according to the random effects model, with statistically significant

480 heterogeneity across studies $\left(I^{2}=95 \%, \mathrm{p}<0.01\right)$. After excluding positive samples that were not

481 confirmed by NT, the pooled MAYV seroprevalence among primates decreased to 4.9 (95\% CI:

$\left.4820.0-15.2 ; I^{2}=96 \% ; \mathrm{p}<0.01\right)$ according to the random effects model. When the analyses were 
483 repeated using the GLMM with logit transformation, seroprevalence estimates for primates

484 decreased to $8.7 \%$ (95\% CI: 3.1-22.0\%) overall and to $0.7 \%$ (95\% CI: $0.0-9.1 \%$ ) when only NT-

485 positive samples were included. Additional meta-analysis results for the various primate genera

486 are presented in S6 and S7 Tables. The seroprevalence for the most frequently sampled primate

487 genera was 32.2\% (95\% CI: 0.0-79.2\%) for the Alouatta genus, $17.8 \%$ (95\% CI: 8.6-28.5\%) for

488 the Callithrix genus, and 3.7\% (95\% CI: 0.0-11.1\%) for the Cebus/Sapajus genus.

490 and forest plots for mammal orders and avian orders are presented in Figs 3 and 4, respectively.

491 When all positive samples were included in the analysis, the highest seroprevalence was

492 observed in the orders Charadriiformes (prevalence: 7.1\%; 95\% CI: 2.2-13.8\%) and Cingulata

493 (prevalence: 3.0\%; 95\% CI: 0.0-24.5\%). When the analysis was repeated using GLMM with

494 logit transformation, the seroprevalence increased to $10.0 \%$ (95\% CI: $2.7-30.8 \%$ ) for the order

495 Cingulata and 9.2\% (95\% CI: 4.4-18.2\%) for the order Charadriiformes. All results of the

496 sensitivity analysis using GLMM with logit transformation are reported in the S5 Table. An

497 additional sensitivity analysis using fixed effects models is presented in the $\mathbf{S 8}$ and S9 Tables.

Table 7. Pooled Prevalence Table (Random effects with Freeman-Tukey double arcsine transformation)

\begin{tabular}{|c|c|c|c|c|c|c|c|c|c|}
\hline Order & $\begin{array}{l}\text { Positives } \\
\text { Included }^{\mathrm{a}}\end{array}$ & $\begin{array}{l}\text { Studies } \\
\text { (n) }\end{array}$ & $\begin{array}{c}\text { Total } \\
\text { (n) }\end{array}$ & $\begin{array}{c}\text { Positive } \\
\text { (n) }\end{array}$ & $\begin{array}{c}\text { Pooled } \\
\text { Prevalence } \\
(\%) \\
\end{array}$ & $95 \% \mathrm{CI}$ & $\begin{array}{l}I^{2} \\
(\%)\end{array}$ & $\tau^{2}$ & $\begin{array}{c}\text { p- } \\
\text { value }\end{array}$ \\
\hline \multicolumn{10}{|c|}{ Mammals } \\
\hline \multirow[t]{2}{*}{ Primate } & $\mathrm{HI}$ and NT & 13 & 897 & 153 & 13.1 & $4.3 ; 25.1$ & 95 & 0.0692 & $<0.01$ \\
\hline & NT only & 13 & 858 & 114 & 4.9 & $0.0 ; 15.2$ & 96 & 0.0851 & $<0.01$ \\
\hline \multirow[t]{2}{*}{ Pilosa } & HI and NT & 7 & 297 & 15 & 0.0 & $0.0 ; 6.6$ & 84 & 0.0338 & $<0.01$ \\
\hline & NT only & 7 & 296 & 14 & 0.0 & $0.0 ; 3.9$ & 82 & 0.0305 & $<0.01$ \\
\hline \multirow[t]{2}{*}{ Rodentia } & $\mathrm{HI}$ and NT & 7 & 1557 & 90 & 1.3 & $0.0 ; 6.5$ & 91 & 0.0160 & $<0.01$ \\
\hline & NT only & 7 & 1486 & 19 & 0.1 & $0.0 ; 3.7$ & 90 & 0.0153 & $<0.01$ \\
\hline \multirow[t]{2}{*}{ Domestic Equids } & $\mathrm{HI}$ and NT & 6 & 1955 & 41 & 1.1 & $0.0 ; 4.5$ & 90 & 0.0085 & $<0.01$ \\
\hline & NT only & 6 & 1940 & 26 & 0.0 & $0.0 ; 1.9$ & 90 & 0.0087 & $<0.01$ \\
\hline \multirow[t]{2}{*}{ Didelphimorphia } & HI and NT & 6 & 369 & 25 & 2.0 & $0.0 ; 7.2$ & 68 & 0.0101 & $<0.01$ \\
\hline & NT only & 6 & 353 & 9 & 0.1 & $0.0 ; 4.2$ & 74 & 0.0141 & $<0.01$ \\
\hline
\end{tabular}




\begin{tabular}{|l|l|c|c|}
\hline Carnivora Order & HI and NT & 5 & 4 \\
\cline { 2 - 4 } & NT only & 5 & 4 \\
\hline \multirow{2}{*}{ Cingulata Order } & HI and NT & 4 & 7 \\
\cline { 2 - 4 } & NT only & 4 & 70 \\
\hline \multirow{2}{*}{ Artiodactyla } & HI and NT & 2 & 26 \\
\cline { 2 - 4 } & NT only & 2 & 26 \\
\hline
\end{tabular}

\begin{tabular}{|l|l}
\hline 40 & \\
\hline 40 & \\
\hline 70 & 6 \\
\hline 70 & 6 \\
\hline 26 & 1 \\
\hline 26 & 1 \\
\hline
\end{tabular}

\begin{tabular}{|l|l|}
\hline 2 & 0. \\
\hline 2 & 0.1 \\
\hline 6 & 3.0 \\
\hline 6 & 3.0 \\
\hline 1 & 2.3 \\
\hline 1 & 2.3 \\
\hline
\end{tabular}

\begin{tabular}{c|c|c}
\hline 0.1 & $0.0 ; 8.1$ & 0 \\
\hline 0.1 & $0.0 ; 8.1$ & 0 \\
\hline 3.0 & $0.0 ; 24.5$ & 35 \\
\hline 3.0 & $0.0 ; 24.5$ & 35 \\
\hline 2.3 & $0.0 ; 20.7$ & 46 \\
\hline 2.3 & $0.0 ; 20.7$ & 46 \\
\hline
\end{tabular}

Birds $^{\mathbf{b}}$

\begin{tabular}{|l|l|c|c|c|c|c|c|c|c|}
\hline Charadriiformes & HI and NT & 3 & 641 & 71 & 7.1 & $2.2 ; 13.8$ & 61 & 0.0045 & 0.08 \\
\hline Passeriformes & HI and NT & 4 & 1166 & 14 & 0.0 & $0.0 ; 0.0$ & 27 & 0.0010 & 0.25 \\
\hline Columbiformes & HI and NT & 4 & 171 & 35 & 2.2 & $0.0 ; 27.1$ & 87 & 0.0591 & $<0.01$ \\
\hline
\end{tabular}

MAYV: Mayaro virus; HI: hemagglutination inhibition; NT: neutralization test; CI: confidence interval

$501{ }^{\text {a }}$ The first analysis (HI and NT) included all positive samples, regardless of test method. A sensitivity analysis was 502 conducted that included only positive samples that were confirmed with NT.

$503{ }^{\mathrm{b}}$ Only one study reporting MAYV positivity in birds used confirmatory NT. Therefore, a sensitivity analysis was not conducted.

Fig 3. Forest plots of mammal orders from meta-analysis of pooled MAYV seroprevalence. Estimates are based on random effects model with Freeman-Tukey double arcsine transformation. All samples that tested MAYV-positive are included, regardless of test method.

Fig 4. Forest plots of avian orders from meta-analysis of pooled MAYV seroprevalence.

\section{MAYV in wild-caught arthropods}

515 caught arthropods. Seventeen $(61 \%)$ of the 28 studies reported at least one arthropod that was

516 positive for MAYV infection. Of the mosquito genera studied, seven were found to be infected

517 with MAYV: Aedes, Culex, Haemagogus, Psorophora, Sabethes, Wyeomyia, and Mansonia. For

518 detailed information on all infected mosquito species, see Table 8. The majority of infected

519 vectors were identified using viral isolation techniques, although three studies reported MAYV

520 positivity using RT-PCR alone. In addition, one study reported isolation of MAYV from an

521 Ixodes tick [91] while another study reported isolation from a Gigantolaelaps mite [52]. 
522 Complete results, including studies that did not detect MAYV in arthropods, are reported in the

\section{S10 Table.}

The geographic distribution of vectors infected with MAYV is presented in Fig 2.

525 MAYV-positive arthropods were identified in four countries overall, including Brazil, Colombia,

526 Panama, and Trinidad. Overall, 15 locations were geo-referenced as points, two locations as

527 ADM1 polygons, two locations as ADM2 polygons, two locations as ADM3 polygons, and two

528 as custom polygons.

Table 8. Evidence of MAYV infection in arthropods

\begin{tabular}{|c|c|c|c|c|}
\hline Genus & Species & Notes & Year & Citation \\
\hline \multirow[t]{9}{*}{ Haemagogus } & \multirow[t]{4}{*}{ Hg. janthinomys } & $\begin{array}{l}\text { Pools of } \mathrm{Hg} \text {. janthinomys yielded nine } \\
\text { isolates by injection into suckling mice }\end{array}$ & 1978 & [19] \\
\hline & & $\begin{array}{l}\text { A pool of two } \mathrm{Hg} \text {. janthinomys yielded } \\
\text { one strain by inoculation into newborn } \\
\text { mice and C6/36 cells and confirmed by } \\
\text { complement fixation and } \\
\text { immunofluorescent assays }\end{array}$ & 2008 & [58] \\
\hline & & $\begin{array}{l}\text { Mayaro virus isolate BeAr505578, } \\
\text { complete genome. GenBank accession } \\
\text { no. KY618129 }\end{array}$ & 1991 & GenBank: KY618129 \\
\hline & & $\begin{array}{l}\text { Mayaro virus isolate BeAr505411. } \\
\text { Genbank accession no. DQ487382 }\end{array}$ & 1991 & [91] \\
\hline & Hg. equinus & $\begin{array}{l}\text { One MAYV isolate detected by viral } \\
\text { culture using Vero cells with } \\
\text { confirmation in microplates. }\end{array}$ & 1973-76 & [72] \\
\hline & Hg. lucifer & $\begin{array}{l}\text { Two MAYV isolates detected by viral } \\
\text { culture using Vero cells with } \\
\text { confirmation in microplates. }\end{array}$ & 1973-76 & [72] \\
\hline & \multirow[t]{3}{*}{ NA } & $\begin{array}{l}\text { Twenty-five isolates reported. No further } \\
\text { information provided. }\end{array}$ & NA & [52] \\
\hline & & $\begin{array}{l}\text { Mayaro virus isolate BeAr350396. } \\
\text { GenBank accession no. DQ487388 }\end{array}$ & 1978 & [91] \\
\hline & & $\begin{array}{l}\text { Complete Genome Sequence of Mayaro } \\
\text { Virus Strain BeAr } 20290 \text {. GenBank } \\
\text { accession no. KT754168. }\end{array}$ & 1960 & [68] \\
\hline \multirow[t]{3}{*}{ Aedes } & \multirow[t]{2}{*}{ Ae. aegypti } & $\begin{array}{l}\text { Two out of } 57(3.5 \%) \text { pools positive by } \\
\text { PCR and isolated in C6/36 cells. }\end{array}$ & 2017 & [79] \\
\hline & & $\begin{array}{l}\text { Four out of } 171(2.3 \%) \text { pools positive by } \\
\text { RT-PCR. One pool yielded an isolate } \\
\text { after inoculation in Vero cells. }\end{array}$ & 2013 & [96] \\
\hline & Ae. serratus & $\begin{array}{l}\text { Addendum to the article states that one } \\
\text { additional MAYV strain was isolated } \\
\text { from Ae. serratus pools. No further }\end{array}$ & 1960 & [75] \\
\hline
\end{tabular}




\begin{tabular}{|c|c|c|c|c|}
\hline & & information provided. & & \\
\hline \multirow[t]{2}{*}{ Mansonia } & \multirow[t]{2}{*}{ M. venezuelensis ${ }^{\mathrm{a}}$} & $\begin{array}{l}\text { MAYV was isolated in baby mice from } \\
\text { a pool of } 49 \text { wild-caught } M . \\
\text { venezuelensis mosquitoes. }\end{array}$ & 1957 & [48] \\
\hline & & $\begin{array}{l}\text { One isolation. No further information } \\
\text { provided. GenBank accession no. } \\
\text { DQ487384. }\end{array}$ & 1957 & {$[52,91]$} \\
\hline \multirow[t]{6}{*}{ Culex } & C. nigripalpus & $\begin{array}{l}\text { One pool out of } 152(0.7 \%) \text { positive by } \\
\text { RT-PCR. }\end{array}$ & 2014-15 & [77] \\
\hline & \multirow[t]{2}{*}{$\begin{array}{l}\text { C. } \\
\text { quinqefasciatus }\end{array}$} & $\begin{array}{l}\text { Twelve out of } 403(3 \%) \text { pools positive } \\
\text { by RT-PCR. One pool was isolated after } \\
\text { inoculation in Vero cells. }\end{array}$ & 2013 & [96] \\
\hline & & $\begin{array}{l}\text { Twelve out of } 179(6.7 \%) \text { pools positive } \\
\text { by RT-PCR and isolation in Vero cells. }\end{array}$ & $2017-18$ & [69] \\
\hline & C. vomerifer & $\begin{array}{l}\text { Wild-caught mosquitoes were allowed to } \\
\text { feed on caged hamsters. The sera of one } \\
\text { hamster produced MAYV antibodies by } \\
\text { HI. }\end{array}$ & 1966 & [71] \\
\hline & \multirow[t]{2}{*}{ NA } & $\begin{array}{l}\text { Mayaro virus strain BeAr757954, } \\
\text { complete genome. GenBank accession } \\
\text { no. KY618130. }\end{array}$ & 2011 & GenBank: KY618130 \\
\hline & & $\begin{array}{l}\text { One isolation. No further information } \\
\text { provided. }\end{array}$ & NA & {$[52]$} \\
\hline \multirow[t]{3}{*}{ Psorophora } & \multirow[t]{2}{*}{ P. ferox } & $\begin{array}{l}\text { A pool of } P \text {. ferox yielded one isolate by } \\
\text { inoculation into Swiss mice. }\end{array}$ & $1959-62$ & [70] \\
\hline & & $\begin{array}{l}\text { Addendum to the article states that five } \\
\text { additional MAYV strains were isolated } \\
\text { from } P \text {. ferox pools. No further } \\
\text { information provided. }\end{array}$ & 1960 & [75] \\
\hline & NA & $\begin{array}{l}\text { Four out of } 748(0.5 \%) \text { pools yielded } \\
\text { strains isolated by inoculation into Swiss } \\
\text { mice from. Pools of } 50 \text { mosquitoes each } \\
\text { were composed of } P \text {. albipes, P. ferox, } \\
\text { or a combination of the two. }\end{array}$ & 1958 & [75] \\
\hline Wyeomyia & NA & $\begin{array}{l}\text { One pool out of } 304(0.3 \%) \text { positive by } \\
\text { RT-PCR. }\end{array}$ & 2006-14 & [64] \\
\hline Sabethes & NA & $\begin{array}{l}\text { Two isolations. No further information } \\
\text { provided }\end{array}$ & NA & [52] \\
\hline Gigantolaelaps & NA & $\begin{array}{l}\text { One isolation. No further information } \\
\text { provided }\end{array}$ & NA & {$[52]$} \\
\hline Ixodes & NA & Genbank accession no. DQ487378 & 1961 & [91] \\
\hline
\end{tabular}

${ }^{\mathrm{a}}$ The mosquito Mansonia venezuelensis is now referred to as Coquillettidia venezuelensis.

\section{Analysis of publication bias}

533 two primate genera. The results of Egger's test did not reveal evidence of publication bias for the

534 included studies. Therefore, the Trim fill technique was not carried out. Funnel plots are

535 presented in S1 and S2 Figures, and results of Egger's test are reported in the S11 Table. 
538 evidence of non-human animal reservoirs and arthropod vectors of MAYV, and the first study to

539 quantitatively analyze the pooled seroprevalence of potential reservoirs. We identified 57

540 studies that assessed MAYV infection in non-human vertebrate animals and arthropods. Overall,

541 the studies found evidence of MAYV infection in 12 wild-caught animal orders and seven

542 arthropod genera across seven Latin American countries and the USA.

544 the orders Primate and Charadriiformes (shorebirds). Several MAYV-positive species were also

545 detected in the orders Rodentia, Didelphimorphia, and Pilosa. Overall, the highest MAYV

546 pooled prevalence occurred in the Primate order. This finding points to the potential role of

547 NHPs as an important reservoir in the MAYV transmission cycle.

The role of NHPs in sylvatic transmission cycles of arboviruses has been demonstrated

549 with varying degrees of evidence [101]. Several arboviruses have been successfully isolated

550 from wild NHPs, including dengue [102], CHIKV [103], and Zika [104] viruses. While isolation

551 of a virus from NHPs is important for establishing the existence of a sylvatic cycle, it is difficult

552 to achieve due to the short duration of viremia [101]. In our review, we identified only one study

553 that successfully isolated MAYV from a NHP [19]. In the absence of viral detection, antibody

554 seroprevalence has been used as evidence of the role of NHPs in sylvatic transmission cycles

$555[105,106]$. Therefore, the high seroprevalence of MAYV among NHPs, including 52\%

556 seropositivity among A. seniculus monkeys in a 1994-95 survey in French Guiana [21], points to

557 the potential importance of NHPs as MAYV reservoirs. Furthermore, Hoch et al. [19] reported 
558 substantial viremia in C. argentata marmosets that were experimentally infected with MAYV

559 and noted that viremia titer was likely sufficient to infect vectors. Due to the high MAYV

560 seroprevalence among marmosets during the Belterra outbreak, the isolation of MAYV from a

561 single $C$. argentata marmoset, and the results of experimental infection studies, the authors

562 concluded that marmosets were likely the amplifying hosts of MAYV.

564 viral isolation from a migrating oriole (Icterus spurius) in Louisiana [60]. Avian species have

565 been implicated as definitive or potential reservoirs of several Alphaviruses, including Sindbis

566 virus [107], Ross River virus [108], and Eastern/Western equine encephalitis virus [109].

567 However, their role in MAYV transmission remains poorly understood. Our systematic review

568 identified seven studies that found MAYV positivity in birds in the orders Passeriformes,

569 Caprimulgiformes, Columbiformes, and Charadriiformes with relatively high seroprevalence

570 reported in several bird species in the latter two orders $[52,53]$. While some have theorized that

571 MAYV has been introduced into certain areas by migratory birds [59], this hypothesis requires

572 further study in order to elucidate the role of birds in MAYV transmission.

Although evidence of MAYV infection was detected in several vertebrate species,

574 identifying the primary non-human animal reservoirs remains a difficult task. The precise

575 definition of a disease "reservoir" has been a source of disagreement [17, 110]. One definition

576 proposed by Haydon et al., (2002) defined a reservoir as "one or more epidemiologically

577 connected populations or environments in which the pathogen can be permanently maintained

578 and from which infection is transmitted to the defined target population" [17]. In addition, in

5792005 Kuno and Chang outlined three basic criteria for the identification of reservoirs including

580 isolation of the virus from the suspected reservoir population, high antibody prevalence in field- 
581 caught animals, and evidence of viremia in laboratory settings, although they posited that

582 definitive identification of a reservoir requires evidence of long-term infection [111]. The role of

583 various non-human vertebrates in the MAYV transmission cycle should be explored further in

584 longitudinal seroprevalence surveys and experimental transmission studies in laboratory settings.

The sylvatic $\mathrm{Hg}$. janthinomys mosquito has long been considered as the primary vector of

586 MAYV. This is in part based on the isolation of MAYV from several pools of $\mathrm{Hg}$. janthinomys

587 mosquitoes in the context of a major MAYV outbreak in Belterra, Brazil in 1978 [19]. Our

588 systematic review also identified several additional mosquito species including Ae. aegypti and

589 Cx. quinqefasciatus with evidence of MAYV infection. A caveat, however, is that the isolation

590 of a virus or detection of viral RNA through PCR is not sufficient to establish that arthropod as a

591 biological vector [112], i.e. involved in the biological transmission of pathogens [111]. The

592 World Health Organization (WHO) established three criteria to define a confirmed vector: (1)

593 viral isolation in the absence of vertebrate blood; (2) biological transmission of the virus in

594 experimental conditions; and (3) presence of certain temporal, geographic and other

595 epidemiological or ecological parameters that allow transmission to occur [112]. Thus, certain

596 arthropods that are capable of ingesting and transmitting a virus may not be established as

597 confirmed vectors if the other parameters are not in place.

599 vector with high MAYV infection rates and transmission potential [22-24, 113]. For example,

600 Long et al., revealed Ae. aegypti to be a capable MAYV vector with a relatively short extrinsic

601 incubation period [22]. Furthermore, MAYV titers in the saliva of Ae. aegypti were similar to

602 other Alphavirus-vector systems including EEEV in Culiseta melanura and VEEV in Ae.

603 albopictus and Ae. taeniorhynchus. In contrast, Cx. quinquefasciatus mosquitoes exhibited low 
604 MAYV infection rates and inability to transmit MAYV in laboratory settings [113]. It is also

605 important to note that the competence of a given vector species to transmit MAYV may be

606 impacted by the MAYV genotype that is present in a given area. In laboratory conditions,

607 genotype L infection rates were significantly higher than genotype D infection rates among Ae.

608 aegypti mosquitoes [113].

609 The spillover of MAYV into urban populations has been a source of concern for Latin

610 American health authorities [114]. The implication is that anthropophilic, urban-dwelling

611 mosquitoes like Ae. aegypti as effective vectors of MAYV would increase the potential for urban

612 MAYV outbreaks [115]. Concerns of urban MAYV transmission were amplified after antibodies

613 to MAYV were discovered in 33 of 631 sera (5.2\%) in the city of Manaus, Brazil in 2007-08

614 [25] although it is unclear if humans can serve as amplification hosts. For example, Long et al.

615 noted that the short duration of MAYV viremia and the relatively low viremic titers in humans

616 reduces the probability of urban spread [22]. Our systematic review identified two recent studies

617 conducted in the city of Cuiaba in which MAYV was isolated from pools of wild-caught Ae.

618 aegypti mosquitoes $[79,96]$. One of these studies also reported vertical transmission of MAYV

619 [79]. This represents another mechanism that may lead to maintenance of the virus in urban

620 mosquito populations. Although Ae. aegypti mosquitoes have not been conclusively implicated

621 as MAYV vectors, the isolation of MAYV from wild-caught Ae. aegypti mosquitoes combined

622 with the evidence of vector competence in laboratory settings [22-24, 113] suggests that MAYV

623 could spill over into an urban cycle. This hypothesis requires further study to explore natural

624 MAYV infection in city-dwelling mosquitoes and additional controlled vector competence

625 studies. 
Our systematic review revealed substantial heterogeneity across included studies, even

627 within animal orders. Heterogeneity may complicate the interpretation of pooled seroprevalence

628 estimates [38]. An additional limitation involves the validity of serological assays used to detect

629 MAYV infection in animals. While plaque reduction NT is considered the "gold standard test"

630 for detecting neutralizing antibodies to MAYV, some of the studies in the review instead relied

631 on the less-specific HI test for antibody detection [101]. Furthermore, antibodies to other

632 alphaviruses in the Semliki Forest serocomplex (e.g., CHIKV) may cross-react in serological

633 tests [116]. Therefore, interpretation of seroprevalence estimates should be done with caution

634 especially in the absence of confirmatory NT. Finally, unpublished data and articles with low

635 quality scores were included in this review due to the paucity of eligible studies. Therefore,

636 readers should consider the heterogeneity of study quality when interpreting the results of pooled

637 seroprevalence estimates

\section{Conclusions}

MAYV is an emerging arbovirus that poses a major threat to human populations in Latin

641 America. In order for public health authorities to effectively design MAYV surveillance and

642 control programs, an understanding of the disease ecology is essential. This systematic review

643 adds to existing knowledge regarding the potential animal reservoirs and arthropod vectors that

644 are involved in the MAYV transmission cycle. These baseline data and maps of MAYV

645 occurrence can direct risk emergence modeling and prediction efforts. Future studies involving

646 experimental infection of primates and other non-human vertebrates are necessary to determine

647 the animal species that may serve as amplifying hosts. Furthermore, additional experimental 
648 transmission studies may provide critical information regarding the potential for Ae. aegypti to

649 facilitate urban spread of MAYV.

\section{Acknowledgments}

651 We would like to thank Dr. Mauro Ramos for his assistance with reviewing Portuguese language

652 articles. ELE is a Scientific researcher of the Consejo de Investigaciones Científicas y

653 Tecnológicas (CONICET) from Argentina.

655 FUNDING STATEMENT: This work was in part conducted by the Infectious Disease

656 Clinical Research Program (IDCRP), a Department of Defense (DoD) program executed by the

657 Uniformed Services University of the Health Sciences (USU) through a cooperative agreement

658 with The Henry M. Jackson Foundation for the Advancement of Military Medicine, Inc. (HJF).

659 This project has been supported with federal funds from the National Institute of Allergy and

660 Infectious Diseases, National Institutes of Health (NIH), under Inter $\square$ Agency Agreement Y1-A1-

6615072 and from the Defense Health Program, U.S. Department of Defense, under award

662 HU0001190002.

663 A.A.'s participation in this work was supported by funding from Armed Forces Health

664 Surveillance Branch - Global Emerging Infections Surveillance (GEIS) Project \#P0044_20_NS

665 and NASA Applied Sciences Program - Health and Air Quality, Grant \#17-HAQ17-0065.

666

667 DISCLAIMER: The contents of this publication are the sole responsibility of the author(s) and 668 do not necessarily reflect the views, opinions or policies of Uniformed Services University of the 669 Health Sciences (USUHS), the Department of Defense (DoD), the Departments of the Army, 
bioRxiv preprint doi: https://doi.org/10.1101/2021.07.28.454243; this version posted July 29, 2021. The copyright holder for this preprint (which

was not certified by peer review) is the author/funder, who has granted bioRxiv a license to display the preprint in perpetuity. It is made available under aCC-BY-NC-ND 4.0 International license.

670 Navy, or Air Force. Mention of trade names, commercial products, or organizations does not

671 imply endorsement by the U.S. Government.

672

673 CONFLICT OF INTEREST: The authors declare no conflicts of interest.

674

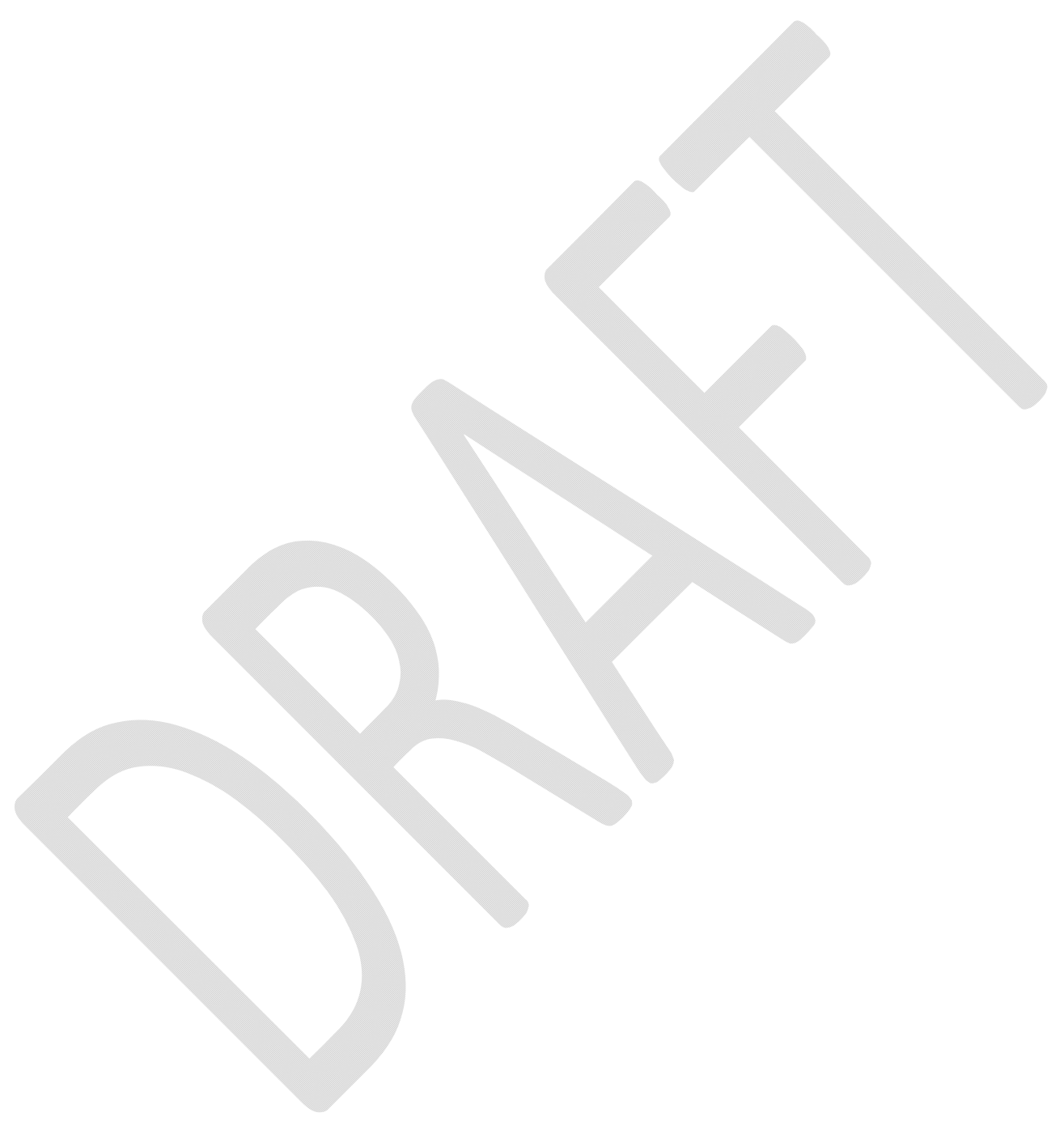




\section{References}

678 1. Anderson CR, Downs WG, Wattley GH, Ahin NW, Reese AA. Mayaro virus: a new 679 human disease agent. II. Isolation from blood of patients in Trinidad, B.W.I. Am J Trop Med 680 Hyg. 1957;6(6):1012-6. Epub 1957/11/01. doi: 10.4269/ajtmh.1957.6.1012. PubMed PMID: 68113487973.

682 2. Suhrbier A, Jaffar-Bandjee MC, Gasque P. Arthritogenic alphaviruses--an overview. Nat 683 Rev Rheumatol. 2012;8(7):420-9. Epub 2012/05/09. doi: 10.1038/nrrheum.2012.64. PubMed 684 PMID: 22565316.

685 3. Pan American Health Organization / World Health Organization. Epidemiological Alert: 686 Mayaro Fever. Washington, D.C.: PAHO/WHO: 2019 May 1, 2019. Report No.

687 4. Causey OR, Maroja OM. Mayaro virus: a new human disease agent. III. Investigation of 688 an epidemic of acute febrile illness on the river Guama in Para, Brazil, and isolation of Mayaro 689 virus as causative agent. Am J Trop Med Hyg. 1957;6(6):1017-23. Epub 1957/11/01. PubMed 690 PMID: 13487974.

691 5. LeDuc JW, Pinheiro FP, Travassos da Rosa AP. An outbreak of Mayaro virus disease in 692 Belterra, Brazil. II. Epidemiology. Am J Trop Med Hyg. 1981;30(3):682-8. Epub 1981/05/01. 693 doi: 10.4269/ajtmh.1981.30.682. PubMed PMID: 6266264.

694 6. Schaeffer M, Gajdusek DC, Lema AB, Eichenwald H. Epidemic jungle fevers among 695 Okinawan colonists in the Bolivian rain forest. I. Epidemiology. Am J Trop Med Hyg. 696 1959;8(3):372-96. doi: 10.4269/ajtmh.1959.8.372.

697 7. Auguste AJ, Liria J, Forrester NL, Giambalvo D, Moncada M, Long KC, et al. 698 Evolutionary and Ecological Characterization of Mayaro Virus Strains Isolated during an 699 Outbreak, Venezuela, 2010. Emerg Infect Dis. 2015;21(10):1742-50. Epub 2015/09/25. doi:

700 10.3201/eid2110.141660. PubMed PMID: 26401714; PubMed Central PMCID: 701 PMCPMC4593426. 
702 8. Forshey BM, Guevara C, Laguna-Torres VA, Cespedes M, Vargas J, Gianella A, et al. 703 Arboviral etiologies of acute febrile illnesses in Western South America, 2000-2007. PLoS Neg1

704 Trop Dis. 2010;4(8):e787. Epub 2010/08/14. doi: 10.1371/journal.pntd.0000787. PubMed

705 PMID: 20706628; PubMed Central PMCID: PMCPMC2919378.

$7069 . \quad$ Jonkers AH, Spence L, Karbaat J. Arbovirus infections in Dutch military personnel

707 stationed in Surinam. Further studies. Trop Geogr Med. 1968;20(3):251-6. Epub 1968/09/01.

708 PubMed PMID: 5683357.

709 10. Navarrete-Espinosa J, Gomez-Dantes H. Arbovirus causales de fiebre hemorrágica en 710 pacientes del Instituto Mexicano del Seguro Social. Rev Med Inst Mex Seguro Soc.

711 2006;44(4):347-53. Epub 2006/08/15. PubMed PMID: 16904038.

712 11. Groot H. Estudios sobre virus transmitidos por artropodos en Colombia. Rev Acad 713 Colomb Cienc. 1964;12(46):191-217. doi: 10.18257/raccefyn.565.

714 12. Talarmin A, Chandler LJ, Kazanji M, de Thoisy B, Debon P, Lelarge J, et al. Mayaro 715 virus fever in French Guiana: isolation, identification, and seroprevalence. Am J Trop Med Hyg. 716 1998;59(3):452-6. Epub 1998/09/28. doi: 10.4269/ajtmh.1998.59.452. PubMed PMID: 9749643.

717 13. Blohm G, Elbadry MA, Mavian C, Stephenson C, Loeb J, White S, et al. Mayaro as a 718 Caribbean traveler: Evidence for multiple introductions and transmission of the virus into Haiti. 719 Int J Infect Dis. 2019;87:151-3. Epub 2019/08/06. doi: 10.1016/j.ijid.2019.07.031. PubMed 720 PMID: 31382049.

721 14. Izurieta RO, Macaluso M, Watts DM, Tesh RB, Guerra B, Cruz LM, et al. Hunting in the 722 Rainforest and Mayaro Virus Infection: An emerging Alphavirus in Ecuador. J Glob Infect Dis. 723 2011;3(4):317-23. Epub 2012/01/10. doi: 10.4103/0974-777x.91049. PubMed PMID: 22223990; 724 PubMed Central PMCID: PMCPMC3249982.

725 15. Plowright RK, Parrish CR, McCallum H, Hudson PJ, Ko AI, Graham AL, et al. Pathways 726 to zoonotic spillover. Nat Rev Microbiol. 2017;15(8):502-10. Epub 2017/05/31. doi:

727 10.1038/nrmicro.2017.45. PubMed PMID: 28555073; PubMed Central PMCID:

728 PMCPMC5791534. 
729 16. Viana M, Mancy R, Biek R, Cleaveland S, Cross PC, Lloyd-Smith JO, et al. Assembling 730 evidence for identifying reservoirs of infection. Trends Ecol Evol. 2014;29(5):270-9. Epub

731 2014/04/15. doi: 10.1016/j.tree.2014.03.002. PubMed PMID: 24726345; PubMed Central

732 PMCID: PMCPMC4007595.

733 17. Haydon DT, Cleaveland S, Taylor LH, Laurenson MK. Identifying reservoirs of

734 infection: a conceptual and practical challenge. Emerg Infect Dis. 2002;8(12):1468-73. Epub

735 2002/12/25. doi: 10.3201/eid0812.010317. PubMed PMID: 12498665; PubMed Central PMCID:

736 PMCPMC2738515.

737 18. Pezzi L, Reusken CB, Weaver SC, Drexler JF, Busch M, LaBeaud AD, et al. GloPID-R

738 report on Chikungunya, O'nyong-nyong and Mayaro virus, part I: Biological diagnostics.

739 Antiviral Res. 2019;166:66-81. Epub 2019/03/25. doi: 10.1016/j.antiviral.2019.03.009. PubMed

$740 \quad$ PMID: 30905821.

741 19. Hoch AL, Peterson NE, LeDuc JW, Pinheiro FP. An outbreak of Mayaro virus disease in

742 Belterra, Brazil. III. Entomological and ecological studies. Am J Trop Med Hyg.

743 1981;30(3):689-98. Epub 1981/05/01. doi: 10.4269/ajtmh.1981.30.689. PubMed PMID:

7446266265.

745 20. Seymour C, Peralta PH, Montgomery GG. Serologic evidence of natural togavirus

746 infections in Panamanian sloths and other vertebrates. Am J Trop Med Hyg. 1983;32(4):854-61.

747 Epub 1983/07/01. doi: 10.4269/ajtmh.1983.32.854. PubMed PMID: 6309027.

748 21. de Thoisy B, Gardon J, Salas RA, Morvan J, Kazanji M. Mayaro virus in wild mammals,

749 French Guiana. Emerg Infect Dis. 2003;9(10):1326-9. Epub 2003/11/12. doi:

750 10.3201/eid0910.030161. PubMed PMID: 14609474; PubMed Central PMCID:

751 PMCPMC3033094.

752 22. Long KC, Ziegler SA, Thangamani S, Hausser NL, Kochel TJ, Higgs S, et al.

753 Experimental transmission of Mayaro virus by Aedes aegypti. Am J Trop Med Hyg.

754 2011;85(4):750-7. Epub 2011/10/07. doi: 10.4269/ajtmh.2011.11-0359. PubMed PMID:

755 21976583; PubMed Central PMCID: PMCPMC3183788. 
756 23. Wiggins K, Eastmond B, Alto BW. Transmission potential of Mayaro virus in Florida 757 Aedes aegypti and Aedes albopictus mosquitoes. Med Vet Entomol. 2018;32(4):436-42. Epub 758 2018/07/15. doi: 10.1111/mve.12322. PubMed PMID: 30006976.

759 24. Brustolin M, Pujhari S, Henderson C, Rasgon J. Emergent viruses and their interactions 760 in Aedes aegypti: Mayaro and zika virus coinfected mosquitoes can successfully transmit both 761 pathogens. Am J Trop Med Hyg. 2019;101(5):50. doi: 10.4269/ajtmh.abstract2019.

762 25. Mourao MP, Bastos Mde S, de Figueiredo RP, Gimaque JB, Galusso Edos S, Kramer 763 VM, et al. Mayaro fever in the city of Manaus, Brazil, 2007-2008. Vector Borne Zoonotic Dis. 764 2012;12(1):42-6. Epub 2011/09/20. doi: 10.1089/vbz.2011.0669. PubMed PMID: 21923266;

765 PubMed Central PMCID: PMCPMC3249893.

766 26. Page MJ, McKenzie JE, Bossuyt PM, Boutron I, Hoffmann TC, Mulrow CD, et al. The 767 PRISMA 2020 statement: An updated guideline for reporting systematic reviews. Int J Surg. 768 2021;88:105906. Epub 2021/04/02. doi: 10.1016/j.ijsu.2021.105906. PubMed PMID: 33789826.

769 27. Clark K, Karsch-Mizrachi I, Lipman DJ, Ostell J, Sayers EW. GenBank. Nucleic Acids 770 Res. 2016;44(D1):D67-D72. Epub 2015/11/20. doi: 10.1093/nar/gkv1276. PubMed PMID: 77126590407.

772 28. Ding H, Gao YM, Deng Y, Lamberton PH, Lu DB. A systematic review and meta773 analysis of the seroprevalence of Toxoplasma gondii in cats in mainland China. Parasit Vectors. 774 2017;10(1):27. Epub 2017/01/15. doi: 10.1186/s13071-017-1970-6. PubMed PMID: 28086987; 775 PubMed Central PMCID: PMCPMC5237326.

776 29. Rodríguez-Monguí E, Cantillo-Barraza O, Prieto-Alvarado FE, Cucunubá ZM.

777 Heterogeneity of Trypanosoma cruzi infection rates in vectors and animal reservoirs in 778 Colombia: a systematic review and meta-analysis. Parasit Vectors. 2019;12(1):308. Epub 779 2019/06/22. doi: 10.1186/s13071-019-3541-5. PubMed PMID: 31221188; PubMed Central 780 PMCID: PMCPMC6585012.

781 30. Guernier V, Goarant C, Benschop J, Lau CL. A systematic review of human and animal 782 leptospirosis in the Pacific Islands reveals pathogen and reservoir diversity. PLoS Negl Trop Dis. 

29758037; PubMed Central PMCID: PMCPMC5967813.

785 31. ESRI. ArcGIS Desktop: Release 10. Redlands, CA: Environmental Systems Research 786 Institute.; 2011.

787 32. Acosta-Ampudia Y, Monsalve DM, Rodriguez Y, Pacheco Y, Anaya JM, Ramirez-

788 Santana C. Mayaro: an emerging viral threat? Emerg Microbes Infect. 2018;7(1):163. Epub 789 2018/09/27. doi: 10.1038/s41426-018-0163-5. PubMed PMID: 30254258; PubMed Central 790 PMCID: PMCPMC6156602.

791 33. Haidich AB. Meta-analysis in medical research. Hippokratia. 2010;14(Suppl 1):29-37.

792 Epub 2011/04/14. PubMed PMID: 21487488; PubMed Central PMCID: PMCPMC3049418.

793 34. Higgins JPT, Thomas J, Chandler J, Cumpston M, Li T, Page MJ, et al. Cochrane

794 Handbook for Systematic Reviews of Interventions version 6.0 Cochrane; 2019. Available from: 795 www.training.cochrane.org/handbook.

796 35. Barendregt JJ, Doi SA, Lee YY, Norman RE, Vos T. Meta-analysis of prevalence. J 797 Epidemiol Community Health. 2013;67(11):974-8. Epub 2013/08/22. doi: 10.1136/jech-2013798 203104. PubMed PMID: 23963506.

799 36. Schwarzer G, Chemaitelly H, Abu-Raddad LJ, Rücker G. Seriously misleading results

800 using inverse of Freeman-Tukey double arcsine transformation in meta-analysis of single

801 proportions. Res Synth Methods. 2019;10(3):476-83. Epub 2019/04/05. doi: 10.1002/jrsm.1348.

802 PubMed PMID: 30945438; PubMed Central PMCID: PMCPMC6767151.

803 37. Warton DI, Hui FK. The arcsine is asinine: the analysis of proportions in ecology.

804 Ecology. 2011;92(1):3-10. Epub 2011/05/13. doi: 10.1890/10-0340.1. PubMed PMID: 80521560670.

806 38. Higgins JPT, Thompson SG, Deeks JJ, Altman DG. Measuring inconsistency in meta807 analyses BMJ. 2003;327:557-60. 
808 39. Quintana DS. From pre-registration to publication: a non-technical primer for conducting

809 a meta-analysis to synthesize correlational data. Front Psychol. 2015;6(1549). doi:

$810 \quad 10.3389 /$ fpsyg.2015.01549.

811 40. Balduzzi S, Rücker G, Schwarzer G. How to perform a meta-analysis with R: a practical

812 tutorial. Evid Based Ment Health. 2019;22(4):153-60. Epub 2019/09/30. doi: 10.1136/ebmental-

813 2019-300117. PubMed PMID: 31563865.

814 41. Egger M, Davey Smith G, Schneider M, Minder C. Bias in meta-analysis detected by a

815 simple, graphical test. BMJ. 1997;315(7109):629-34. Epub 1997/10/06. doi:

816 10.1136/bmj.315.7109.629. PubMed PMID: 9310563; PubMed Central PMCID:

817 PMCPMC2127453.

818 42. Duval S, Tweedie R. Trim and fill: A simple funnel-plot-based method of testing and

819 adjusting for publication bias in meta-analysis. Biometrics. 2000;56(2):455-63. Epub

820 2000/07/06. doi: 10.1111/j.0006-341x.2000.00455.x. PubMed PMID: 10877304.

821 43. Messina JP, Brady OJ, Pigott DM, Brownstein JS, Hoen AG, Hay SI. A global

822 compendium of human dengue virus occurrence. Sci Data. 2014;1:140004. Epub 2014/01/01.

823 doi: 10.1038/sdata.2014.4. PubMed PMID: 25977762; PubMed Central PMCID:

824 PMCPMC4322574.

825 44. Pigott DM, Golding N, Messina JP, Battle KE, Duda KA, Balard Y, et al. Global 826 database of leishmaniasis occurrence locations, 1960-2012. Sci Data. 2014;1:140036. Epub

827 2014/01/01. doi: 10.1038/sdata.2014.36. PubMed PMID: 25984344; PubMed Central PMCID:

828 PMCPMC4432653.

829 45. Runfola D, Anderson A, Baier H, Crittenden M, Dowker E, Fuhrig S, et al.

830 geoBoundaries: A global database of political administrative boundaries. PloS One.

831 2020;15(4):e0231866. Epub 2020/04/25. doi: 10.1371/journal.pone.0231866. PubMed PMID:

832 32330167; PubMed Central PMCID: PMCPMC7182183 Allen Hamilton, and Deloitte,

833 respectively. This does not alter our adherence to PLOS ONE policies on sharing data and

834 materials. 
835 46. de Thoisy B, Vogel I, Reynes JM, Pouliquen JF, Carme B, Kazanji M, et al. Health

836 evaluation of translocated free-ranging primates in French Guiana. Am J Primatol. 2001;54(1):1-

837 16. Epub 2001/05/01. doi: 10.1002/ajp.1008. PubMed PMID: 11329164.

838 47. Aitken TH, Downs WG, Anderson CR, Spence L, Casals J. Mayaro virus isolated from a 839 Trinidadian mosquito, Mansonia venezuelensis. Science (New York, NY). 1960;131(3405):986.

840 Epub 1960/04/01. doi: 10.1126/science.131.3405.986. PubMed PMID: 13792204.

841 48. Aitken TH, Spence L, Jonkers AH, Downs WG. A 10-year survey of Trinidadian 842 arthropods for natural virus infections (1953-1963). J Med Entomol. 1969;6(2):207-15. Epub

843 1969/05/01. doi: 10.1093/jmedent/6.2.207. PubMed PMID: 5807863.

844 49. Batista PM, Andreotti R, Almeida PS, Marques AC, Rodrigues SG, Chiang JO, et al.

845 Detection of arboviruses of public health interest in free-living New World primates (Sapajus

846 spp.; Alouatta caraya) captured in Mato Grosso do Sul, Brazil. Rev Soc Bras Med Trop.

847 2013;46(6):684-90. Epub 2014/01/30. doi: 10.1590/0037-8682-0181-2013. PubMed PMID:

84824474008.

849 50. Paulo M, Renato A, Da Carneiro Rocha T, Eliane C, Navarro da Silva M. Serosurvey of 850 arbovirus in free-living non-human primates (Sapajus spp.) in Brazil. J Environ Anal Chem.

$851 \quad 2015 ; 2(155): 2380-91.1000155$.

852 51. Woodall JP. Virus Research in Amazonia. Atas do Simpósio Sobre a Biota Amazônica; 853 Para, Brazil1967. p. 31-63.

854 52. Taylor RM. Catalogue of arthropod-borne viruses of the world: a collection of data on 855 registered arthropod-borne animal viruses: US Public Health Service; 1967.

856 53. Araujo FAA, Wada MY, da Silva EV, Cavalcante GC, Magalhaes VS, de Andrade Filho $857 \mathrm{GV}$, et al. Primeiro inquérito sorológico em aves migratórias e nativas do Parque Nacional da 858 Lagoa do Peixe/RS para detecção do vírus do Nilo Ocidental. In: Ministério da Saúde Secretaria 859 de Vigilância em Saúde, editor. Boletim Eletrônico Epidemiologico, 2003.

860 54. Araújo FAA, Vianna RdST, Andrade Filho GVd, Melhado DL, Todeschini B, Cavalcante 861 e Cavalcanti G, et al. Segundo inquérito sorológico em aves migratórias e residentes do parque 45 
862 nacional da Lagoa do Peixe/RS para detecção do vírus da Febre da Febre do Nilo Ocidental e

863 outros vírus. In: Ministério da Saúde Secretaria de Vigilância em Saúde, editor. Boletim

864 Eletrônico Epidemiologico, 2004.

865 55. Araújo FAA, Vianna RdST, Wada MY, Silva ÉVd, Doretto L, Cavalcante GCe, et al.

866 Inquérito sorológico em aves migratórias e residentes de Galinhos/RN para detecção do vírus da

867 Febre do Nilo Ocidental e outros vírus. In: Ministério da Saúde Secretaria de Vigilância em

868 Saúde, editor. Boletim Eletrônico Epidemiológico, 2004.

869 56. Araujo FAA, Lima PC, Andrade MA, de Sá Jayme V, Ramos DG, Da Silveira SL.

870 Soroprevalência de anticorpos "anti-arbovírus" de importância em saúde pública em aves

871 selvagens, Brasil-2007 e 2008. Ciênc Anim Brasil. 2012;13(1):115-23. doi:

$872 \quad 10.5216 /$ cab.v13i1.16834.

873 57. Araujo FAA, Andrade MA, Jayme VS, Santos AL, Roman APM, Ramos DG, et al.

874 Anticorpos antialfavírus detectados em equinos durante diferentes epizootias de encefalite

875 equina, Paraíba, 2009. Rev Bras Ciênc Vet. 2012;19(1):80-5. doi: 10.4322/rbcv.2014.086.

876 58. Azevedo RS, Silva EV, Carvalho VL, Rodrigues SG, Neto JPN, Monteiro HA, et al.

877 Mayaro fever virus, Brazilian amazon. Emerg Infect Dis. 2009;15(11):1830. doi:

$878 \quad$ 10.3201/eid1511.090461.

879 59. Batista PM, Andreotti R, Chiang JO, Ferreira MS, Vasconcelos PF. Seroepidemiological 880 monitoring in sentinel animals and vectors as part of arbovirus surveillance in the state of Mato

881 Grosso do Sul, Brazil. Rev Soc Bras Med Trop. 2012;45(2):168-73. Epub 2012/04/27. doi:

882 10.1590/s0037-86822012000200006. PubMed PMID: 22534986.

883 60. Calisher CH, Gutierrez E, Maness KS, Lord RD. Isolation of Mayaro virus from a

884 migrating bird captured in Louisiana in 1967. Bull Pan Am Health Organ. 1974;8(3):243-8. Epub

885 1974/01/01. PubMed PMID: 4418030.

886 61. Carrera JP, Cucunubá ZM, Neira K, Lambert B, Pittí Y, Liscano J, et al. Endemic and 887 Epidemic Human Alphavirus Infections in Eastern Panama: An Analysis of Population-Based 

0408. PubMed PMID: 33124532.

890 62. Casseb AdR. Soroprevalência de anticorpos e padronização do teste ELISA sanduíche

891 indireto para 19 tipos de arbovírus em herbívoros domésticos [Ph.D. Thesis]. Belém:

892 Universidade Federal do Pará; 2010. Available from:

893 http://repositorio.ufpa.br/jspui/handle/2011/4760.

894 63. Casseb AdR, Brito TC, Silva MRMd, Chiang JO, Martins LC, Silva SPd, et al.

895 Prevalence of antibodies to equine alphaviruses in the State of Pará, Brazil. Arq Inst Biol.

896 2016;83. doi: 10.1590/1808-1657000202014.

897 64. Catenacci LS. Abordagem one health para vigilância de arbovirus na Mata Atlântica do

898 sul da Bahia, Brasil. [Ph.D. Thesis]. Ananindeua: Instituto Evandro Chagas; 2017. Available

899 from: https://patua.iec.gov.br/handle/iec/3073.

900 65. Cruz ACR, Prazeres AdSCd, Gama EC, Lima MFd, Azevedo RdSS, Casseb LMN, et al.

901 Vigilância sorológica para arbovírus em Juruti, Pará, Brasil. Cadernos de saude publica.

$902 \quad 2009 ; 25(11): 2517-23$.

903 66. Degallier N, Travassos da Rosa AP, Vasconcelos PFC, Hervé JP, Sa Filho GC, Travassos

904 da Rosa JFS, et al. Modifications of arbovirus transmission in relation to construction of dams in

905 Brazilian Amazonia Journal of the Brazilian Association for the Advancement of Science.

$9061992 ; 44$.

907 67. Diaz LA, Diaz Mdel P, Almiron WR, Contigiani MS. Infection by UNA virus

908 (Alphavirus; Togaviridae) and risk factor analysis in black howler monkeys (Alouatta caraya)

909 from Paraguay and Argentina. Trans R Soc Trop Med Hyg. 2007;101(10):1039-41. Epub

910 2007/07/31. doi: 10.1016/j.trstmh.2007.04.009. PubMed PMID: 17658571.

911 68. Esposito DL, da Fonseca BA. Complete Genome Sequence of Mayaro Virus

912 (Togaviridae, Alphavirus) Strain BeAr 20290 from Brazil. Genome Announc. 2015;3(6). Epub

913 2015/12/19. doi: 10.1128/genomeA.01372-15. PubMed PMID: 26679574; PubMed Central

914 PMCID: PMCPMC4683219. 
915 69. da Silva Ferreira R, de Toni Aquino da Cruz LC, Souza VJ, da Silva Neves NA, de Souza

916 VC, Filho LCF, et al. Insect-specific viruses and arboviruses in adult male culicids from

917 Midwestern Brazil. Infect Genet Evol. 2020:104561. Epub 2020/09/23. doi:

918 10.1016/j.meegid.2020.104561. PubMed PMID: 32961364.

919 70. Galindo P, Srihongse S, De Rodaniche E, Grayson MA. An ecological survey for

920 arboviruses in Almirante, Panama, 1959-1962. Am J Trop Med Hyg. 1966;15(3):385-400. Epub

921 1966/05/01. doi: 10.4269/ajtmh.1966.15.385. PubMed PMID: 4380043.

922 71. Galindo P, Srihongse S. Transmission of arboviruses to hamsters by the bite of naturally

923 infected Culex (Melanoconion) mosquitoes. Am J Trop Med Hyg. 1967;16(4):525-30. Epub

924 1967/07/01. doi: 10.4269/ajtmh.1967.16.525. PubMed PMID: 4952151.

925 72. Galindo P, Adames A, Peralta P, Johnson C, Read R. Impacto de la hidroeléctrica de

926 Bayano en la transmisión de arbovirus. Rev Med Pan. 1983;8:89-134.

927 73. Gibrail MM. Detecção de anticorpos para arbovirus em primatas não humanos no

928 município de Goiânia, Goiás [M.Sc. Thesis]. Goiânia: Universidade Federal de Goiás; 2015.

929 Available from: https://repositorio.bc.ufg.br/tede/handle/tede/5552.

930 74. Gomes FA, Jansen AM, Machado RZ, Jesus Pena HF, Fumagalli MJ, Silva A, et al.

931 Serological evidence of arboviruses and coccidia infecting horses in the Amazonian region of

932 Brazil. PloS One. 2019;14(12):e0225895. Epub 2019/12/13. doi: 10.1371/journal.pone.0225895.

933 PubMed PMID: 31830142.

934 75. Groot H, Morales A, Vidales H. Virus isolations from forest mosquitoes in San Vicente

935 de Chucuri, Colombia. Am J Trop Med Hyg. 1961;10:397-402. Epub 1961/05/01. doi:

936 10.4269/ajtmh.1961.10.397. PubMed PMID: 13708940.

937 76. Henriques DA. Caracterização molecular de arbovírus isolados da fauna diptera

938 nematocera do Estado de Rondônia (Amazônia ocidental brasileira) [Ph.D. Thesis]. São Paulo:

939 Universidade de São Paulo; 2008. Available from:

940 https://teses.usp.br/teses/disponiveis/42/42132/tde-27032009-124003/pt-br.php. 
941 77. Kubiszeski JR. Arboviroses emergentes no município de Sinop-MT: pesquisa de vetores

942 [Ph.D. Thesis]. Sinop: Universidade Federal de Mato Grosso; 2016. Available from:

943 https://teses.usp.br/teses/disponiveis/42/42132/tde-27032009-124003/pt-br.php.

944 78. Laroque PO, Valença-Montenegro MM, Ferreira DRA, Chiang JO, Cordeiro MT,

945 Vasconcelos PFC, et al. Levantamento soroepidemiológico para arbovírus em macaco-prego-

946 galego (Cebus flavius) de vida livre no estado da Paraíba e em macaco-prego (Cebus libidinosus)

947 de cativeiro do nordeste do Brasil. Pesq Vet Bras. 2014;34:462-8.

948 79. Maia LMS, Bezerra MCF, Costa MCS, Souza EM, Oliveira MEB, Ribeiro ALM, et al.

949 Natural vertical infection by dengue virus serotype 4, Zika virus and Mayaro virus in Aedes

950 (Stegomyia) aegypti and Aedes (Stegomyia) albopictus. Med Vet Entomol. 2019;33(3):437-42.

951 Epub 2019/02/19. doi: 10.1111/mve.12369. PubMed PMID: 30776139.

952 80. Martinez D, Hernandez C, Munoz M, Armesto Y, Cuervo A, Ramirez JD. Identification

953 of Aedes (Diptera: Culicidae) Species and Arboviruses Circulating in Arauca, Eastern Colombia.

954 Front Ecol Evol. 2020;8. doi: 10.3389/fevo.2020.602190. PubMed PMID:

955 WOS:000596835300001.

956 81. Medlin S, Deardorff ER, Hanley CS, Vergneau-Grosset C, Siudak-Campfield A, Dallwig

957 R, et al. Serosurvey of Selected Arboviral Pathogens in Free-Ranging, Two-Toed Sloths

958 (Choloepus Hoffmanni) and Three-Toed Sloths (Bradypus Variegatus) In Costa Rica, 2005-07. J

959 Wildl Dis. 2016;52(4):883-92. Epub 2016/08/02. doi: 10.7589/2015-02-040. PubMed PMID:

$96027479900 ;$ PubMed Central PMCID: PMCPMC5189659.

961 82. Medina G, Garzaro DJ, Barrios M, Auguste AJ, Weaver SC, Pujol FH. Genetic diversity

962 of Venezuelan alphaviruses and circulation of a Venezuelan equine encephalitis virus subtype

963 IAB strain during an interepizootic period. Am J Trop Med Hyg. 2015;93(1):7-10. Epub

964 2015/05/06. doi: 10.4269/ajtmh.14-0543. PubMed PMID: 25940191; PubMed Central PMCID:

965 PMCPMC4497907.

966 83. Moreira-Soto A, Carneiro ID, Fischer C, Feldmann M, Kummerer BM, Silva NS, et al.

967 Limited Evidence for Infection of Urban and Peri-urban Nonhuman Primates with Zika and 
968 Chikungunya Viruses in Brazil. mSphere. 2018;3(1). doi: 10.1128/mSphere.00523-17. PubMed

969 PMID: WOS:000425277500024.

970 84. Nunes MR, Barbosa TF, Casseb LM, Nunes Neto JP, Segura Nde O, Monteiro HA, et al.

971 Eco-epidemiologia dos arbovirus na area de influencia da rodovia Cuiaba-Santarem (BR 163),

972 Estado do Para, Brasil. Cad Saude Publica. 2009;25(12):2583-602. Epub 2010/03/02. doi:

973 10.1590/s0102-311x2009001200006. PubMed PMID: 20191150.

974 85. Pauvolid-Correa A, Tavares FN, Costa EV, Burlandy FM, Murta M, Pellegrin AO, et al.

975 Serologic evidence of the recent circulation of Saint Louis encephalitis virus and high prevalence

976 of equine encephalitis viruses in horses in the Nhecolandia sub-region in South Pantanal,

977 Central-West Brazil. Mem Inst Oswaldo Cruz. 2010;105(6):829-33. Epub 2010/10/15. doi:

978 10.1590/s0074-02762010000600017. PubMed PMID: 20945001.

979 86. Pauvolid-Correa A, Juliano RS, Campos Z, Velez J, Nogueira RM, Komar N.

980 Neutralising antibodies for Mayaro virus in Pantanal, Brazil. Mem Inst Oswaldo Cruz.

981 2015;110(1):125-33. Epub 2015/03/06. doi: 10.1590/0074-02760140383. PubMed PMID:

982 25742272; PubMed Central PMCID: PMCPMC4371226.

983 87. Pauvolid-Correa A. Estudo sobre arbovírus em populações de eqüinos e artrópodes na

984 sub-região da Nhecolândia no Pantanal de Mato Grosso do Sul [M.Sc. Thesis]. Rio de Janeiro:

985 Fundação Oswaldo Cruz; 2008. Available from: https://www.arca.fiocruz.br/handle/icict/21142.

986 88. Perez JG, Carrera JP, Serrano E, Pitti Y, Maguina JL, Mentaberre G, et al. Serologic

987 Evidence of Zoonotic Alphaviruses in Humans from an Indigenous Community in the Peruvian

988 Amazon. Am J Trop Med Hyg. 2019. Epub 2019/10/02. doi: 10.4269/ajtmh.18-0850. PubMed

989 PMID: 31571566.

990 89. Pinheiro FP, Bensabath G, Andrade AH, Lins ZC, Fraihi H, Tang AT, et al. Infectious

991 diseases along Brazil's Trans-Amazon Highway: surveillance and research. Bull Pan Am Health

992 Organ. 1974;8(111).

993 90. Pinheiro GG, Rocha MN, de Oliveira MA, Moreira LA, Andrade JD. Detection of

994 Yellow Fever Virus in Sylvatic Mosquitoes during Disease Outbreaks of 2017-2018 in Minas 
995 Gerais State, Brazil. Insects. 2019;10(5). doi: 10.3390/insects10050136. PubMed PMID:

996 WOS:000476846800018.

997 91. Powers AM, Aguilar PV, Chandler LJ, Brault AC, Meakins TA, Watts D, et al. Genetic 998 relationships among Mayaro and Una viruses suggest distinct patterns of transmission. Am J

999 Trop Med Hyg. 2006;75(3):461-9. Epub 2006/09/14. PubMed PMID: 16968922.

1000 92. Price JL. Serological evidence of infection of Tacaribe virus and arboviruses in

1001 Trinidadian bats. Am J Trop Med Hyg. 1978;27(1 Pt 1):162-7. Epub 1978/01/01. doi:

1002 10.4269/ajtmh.1978.27.162. PubMed PMID: 204207.

1003 93. Ragan IK, Hartwig A, Bowen RA. Cold blood: Reptiles and amphibians as reservoir and 1004 over wintering hosts for arboviruses. Am J Trop Med Hyg. 2019;101(5):261. doi:

1005 10.4269/ajtmh.abstract2019.

1006 94. Sanmartín C, Mackenzie RB, Trapido H, Barreto P, Mullenax CH, Gutiérrez E, et al.

1007 Encefalitis equina venezolana en Colombia, 1967. Bol Oficina Sanit Panam. 1973;74(2):108-37.

1008 Epub 1973/02/01. PubMed PMID: 4265714.

1009 95. Scherer WF, Madalengoitia J, Flores W, Acosta M. The first isolations of eastern 1010 encephalitis, group C, and Guama group arboviruses from the Peruvian Amazon region of 1011 western South America. Bull Pan Am Health Organ. 1975;9(1):19-26. Epub 1975/01/01. 1012 PubMed PMID: 238693.

1013 96. Serra OP, Cardoso BF, Ribeiro AL, Santos FA, Slhessarenko RD. Mayaro virus and 1014 dengue virus 1 and 4 natural infection in culicids from Cuiaba, state of Mato Grosso, Brazil.

1015 Mem Inst Oswaldo Cruz. 2016;111(1):20-9. Epub 2016/01/20. doi: 10.1590/0074-02760150270.

1016 PubMed PMID: 26784852; PubMed Central PMCID: PMCPMC4727432.

1017 97. Silva JWP. Aspectos ecológicos de vetores putativos do Vírus Mayaro e Vírus Oropuche 1018 em estratificação vertical e horizontal em ambientes florestais e antropizados em uma 1019 comunidade rural no Amazonas [M.Sc. Thesis]. Manaus, AM: Oswaldo Cruz Foundation, 1020 Instituto Leônidas and Maria Deane; 2017. Available from:

1021 https://www.arca.fiocruz.br/handle/icict/23337. 
1022 98. Srihongse S, Galindo P, Eldridge BF. A survey to assess potential human disease hazards 1023 along proposed sea level canal routes in Panama and Colombia. V. Arbovirus infection in non 1024 human vertebrates. Mil Med. 1974;139(6):449-53.

1025 99. Tauro LB, Cardoso CW, Souza RL, Nascimento LC, Santos DRD, Campos GS, et al. A 1026 localized outbreak of Chikungunya virus in Salvador, Bahia, Brazil. Mem Inst Oswaldo Cruz.

1027 2019;114:e180597. Epub 2019/03/08. doi: 10.1590/0074-02760180597. PubMed PMID:

1028 30843962; PubMed Central PMCID: PMCPMC6396974.

1029 100. Turell MJ, Gozalo AS, Guevara C, Schoeler GB, Carbajal F, Lopez-Sifuentes VM, et al. 1030 Lack of Evidence of Sylvatic Transmission of Dengue Viruses in the Amazon Rainforest Near 1031 Iquitos, Peru. Vector Borne Zoonotic Dis. 2019;19(9):685-9. Epub 2019/04/10. doi:

1032 10.1089/vbz.2018.2408. PubMed PMID: 30964397; PubMed Central PMCID:

1033 PMCPMC6716187.

1034 101. Valentine MJ, Murdock CC, Kelly PJ. Sylvatic cycles of arboviruses in non-human 1035 primates. Parasit Vectors. 2019;12(1):463. Epub 2019/10/04. doi: 10.1186/s13071-019-3732-0. 1036 PubMed PMID: 31578140; PubMed Central PMCID: PMCPMC6775655.

1037 102. Cornet M, Saluzzo JF, Hervy JP, Digoutte JP, Germain M, Chauvancy MF. Dengue 2 au 1038 Sénégal oriental: une pousse épizootique en milieu selvatique; isolements du virus à partir de 1039 moustiques et d'un singe et considérations épidémiologiques. Cah Orstom Ser Ent Med Parasitol. $1040 \quad 1984 ; 22: 313-23$.

1041 103. Diallo M, Thonnon J, Traore-Lamizana M, Fontenille D. Vectors of Chikungunya virus 1042 in Senegal: current data and transmission cycles. Am J Trop Med Hyg. 1999;60(2):281-6. Epub 1043 1999/03/11. doi: 10.4269/ajtmh.1999.60.281. PubMed PMID: 10072152.

1044 104. Dick GW, Kitchen SF, Haddow AJ. Zika virus. I. Isolations and serological specificity. 1045 Trans R Soc Trop Med Hyg. 1952;46(5):509-20. Epub 1952/09/01. doi: 10.1016/00351046 9203(52)90042-4. PubMed PMID: 12995440.

1047 105. Althouse BM, Guerbois M, Cummings DAT, Diop OM, Faye O, Faye A, et al. Role of 1048 monkeys in the sylvatic cycle of chikungunya virus in Senegal. Nat Commun. 2018;9(1):1046. 
1049 Epub 2018/03/15. doi: 10.1038/s41467-018-03332-7. PubMed PMID: 29535306; PubMed 1050 Central PMCID: PMCPMC5849707.

1051 106. Kading RC, Borland EM, Cranfield M, Powers AM. Prevalence of antibodies to

1052 alphaviruses and flaviviruses in free-ranging game animals and nonhuman primates in the greater

1053 Congo basin. J Wildl Dis. 2013;49(3):587-99. Epub 2013/06/20. doi: 10.7589/2012-08-212.

1054 PubMed PMID: 23778608.

1055 107. Lundström JO, Lindström KM, Olsen B, Dufva R, Krakower DS. Prevalence of sindbis 1056 virus neutralizing antibodies among Swedish passerines indicates that thrushes are the main 1057 amplifying hosts. J Med Entomol. 2001;38(2):289-97. Epub 2001/04/12. doi: 10.1603/00221058 2585-38.2.289. PubMed PMID: 11296837.

1059 108. Stephenson EB, Peel AJ, Reid SA, Jansen CC, McCallum H. The non-human reservoirs 1060 of Ross River virus: a systematic review of the evidence. Parasit Vectors. 2018;11(1):188. Epub 1061 2018/03/21. doi: 10.1186/s13071-018-2733-8. PubMed PMID: 29554936; PubMed Central 1062 PMCID: PMCPMC5859426.

1063 109. Barba M, Fairbanks EL, Daly JM. Equine viral encephalitis: prevalence, impact, and 1064 management strategies. Vet Med (Auckl). 2019;10:99-110. Epub 2019/09/10. doi:

1065 10.2147/vmrr.S168227. PubMed PMID: 31497528; PubMed Central PMCID:

1066 PMCPMC6689664.

1067 110. Kuno G, Mackenzie JS, Junglen S, Hubálek Z, Plyusnin A, Gubler DJ. Vertebrate 1068 reservoirs of arboviruses: myth, synonym of amplifier, or reality? Viruses. 2017;9(7):185.

1069 111. Kuno G, Chang GJ. Biological transmission of arboviruses: reexamination of and new 1070 insights into components, mechanisms, and unique traits as well as their evolutionary trends. 1071 Clin Microbiol Rev. 2005;18(4):608-37. Epub 2005/10/15. doi: 10.1128/cmr.18.4.608-637.2005. 1072 PubMed PMID: 16223950; PubMed Central PMCID: PMCPMC1265912.

1073 112. World Health Organization Scientific Group. Arthropod-borne and rodent-borne viral 1074 diseases. Geneva, Switzerland: World Health Organization, 1985. 
1075 113. Pereira TN, Carvalho FD, De Mendonça SF, Rocha MN, Moreira LA. Vector

1076 competence of Aedes aegypti, Aedes albopictus, and Culex quinquefasciatus mosquitoes for

1077 Mayaro virus. PLoS Negl Trop Dis. 2020;14(4):e0007518. Epub 2020/04/15. doi:

1078 10.1371/journal.pntd.0007518. PubMed PMID: 32287269; PubMed Central PMCID:

1079 PMCPMC7182273.

1080 114. Mackay IM, Arden KE. Mayaro virus: a forest virus primed for a trip to the city?

1081 Microbes Infect. 2016;18(12):724-34. Epub 2016/12/19. doi: 10.1016/j.micinf.2016.10.007.

1082 PubMed PMID: 27989728.

1083 115. Figueiredo MLGd, Figueiredo LTM. Emerging alphaviruses in the Americas:

1084 Chikungunya and Mayaro. Revista da Sociedade Brasileira de Medicina Tropical.

1085 2014;47(6):677-83. doi: 10.1590/0037-8682-0246-2014.

1086 116. Hassing RJ, Leparc-Goffart I, Tolou H, van Doornum G, van Genderen PJ. Cross-

1087 reactivity of antibodies to viruses belonging to the Semliki forest serocomplex. Eurosurveillance.

$1088 \quad 2010 ; 15(23)$.

1089

Supplementary Materials

1090 S1 Table. PRISMA Checklist

1091 S2 Table. MAYV positivity by taxa of wild mammals in included studies

1092 S3 Table. MAYV positivity by taxa of wild birds in included studies

1093 S4 Table. MAYV positivity in domestic or sentinel animals studied

1094 S5 Table. Pooled Prevalence Table (Random effects using GLMM with logit

1095 transformation)

1096 S6 Table. Primate Genera Pooled Prevalence Table (Random effects with Freeman-Tukey

1097 double arcsine transformation)

1098 S7 Table. Primate Genera Pooled Prevalence Table (Random effects using GLMM with

1099 logit transformation)

1100 S8 Table. Pooled Prevalence Table (Fixed effects with Freeman-Tukey double arcsine

1101 transformation)

1102 S9 Table. Pooled Prevalence Table (Fixed effects using GLMM with logit transformation)

1103 S1 Fig. Funnel plots for estimates of MAYV seroprevalence in non-human animal

1104 reservoirs

1105 S2 Fig: Funnel plots for estimates of MAYV seroprevalence in non-human primate genera

1106 S10 Table. Complete arthropod results by genus

1107 S11 Table. Egger's test for publication bias 
Records identified through initial database search: PubMed, Embase, Web of Science, SciELO

$$
\text { (n=893) }
$$

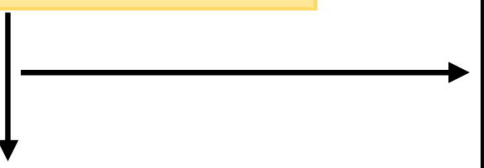

Records screened for title and abstract from initial search $(n=394)$

Additional records screened from secondary search: preprint servers, dissertations, PAHO, Gideon, ASTMH, GenBank $(n=630)$

Full text articles/records assessed for eligibility from initial search $(n=40)$ and secondary search $(n=47)$

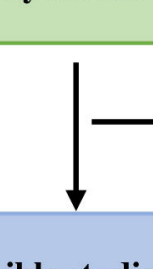

Total eligible studies $(n=57)^{a}$

Animal studies $(\mathrm{n}=39)$

Arthropod studies ( $\mathrm{n}=29)$
Records excluded ( $n=354)$

Records excluded ( $\mathrm{n}=\mathbf{5 8 3})$

\section{Records excluded $(n=30)$ \\ Reasons:}

Did not meet inclusion criteria $(\mathrm{n}=21)$

Duplicate data $(\mathrm{n}=8)$

Not available $(\mathrm{n}=1)$

Article erratum $(n=1)$

Studies included in qualitative analysis $(\mathbf{n}=57)$

Studies included in quantitative metaanalysis $(n=24)$ 


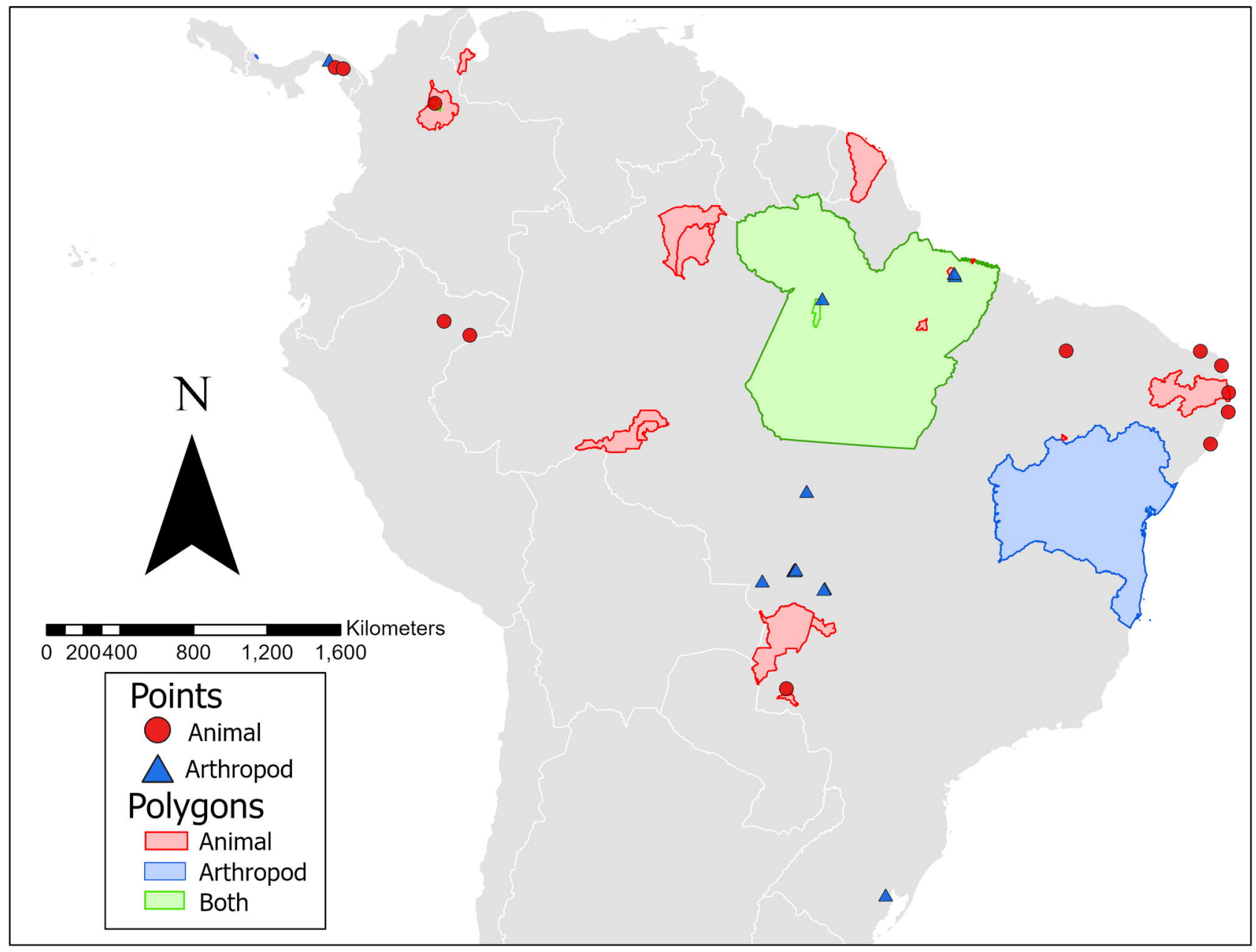


Passeriformes

\begin{tabular}{|c|c|c|c|c|c|c|c|c|c|c|}
\hline Study & Events & Total & & & & & & Proportion & $95 \%-\mathrm{Cl}$ & Weight \\
\hline Hoch, 1981 & 14 & 1003 & + & & & & & 0.01 & {$[0.01 ; 0.02]$} & $65.9 \%$ \\
\hline Cruz, 2009 & 0 & 5 & 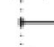 & & & & & 0.00 & {$[0.00 ; 0.52]$} & $1.8 \%$ \\
\hline Sanmartin, 1973 & 0 & 156 & 분 & & & & & 0.00 & {$[0.00 ; 0.02]$} & $31.5 \%$ \\
\hline Araujo, 2012 & 0 & 2 & & & & & & 0.00 & {$[0.00 ; 0.84]$} & $0.8 \%$ \\
\hline Random effects mode & & 1166 & $\mathrm{i}$ & & & & & 0.00 & {$[0.00 ; 0.00]$} & $100.0 \%$ \\
\hline \multirow{2}{*}{ Heterogeneity: $I^{2}=27 \%$} & $2=0.0010$ & $p=0.25$ & \ulcorner & 1 & 1 & 1 & 1 & ᄀ & & \\
\hline & & & 0 & 0.2 & 0.4 & 0.6 & 0.8 & 1 & & \\
\hline
\end{tabular}

\section{Columbiformes}

\section{Study}

Hoch, 1981

Taylor, 1967

Cruz, 2009

Sanmartin, 1973

Random effects model

Heterogeneity: $I^{2}=87 \%, \tau^{2}=0.0591, p<0.01$

Events Total

171

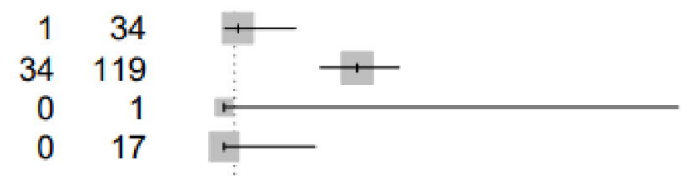

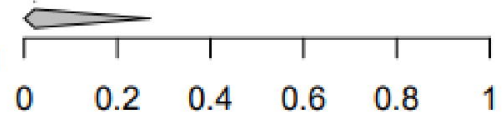

Proportion

$95 \%-\mathrm{Cl}$ Weight

$0.03[0.00 ; 0.15] \quad 30.5 \%$

$0.29[0.21 ; 0.38] \quad 33.0 \%$

$0.00[0.00 ; 0.98] \quad 9.0 \%$

$0.00[0.00 ; 0.20] \quad 27.5 \%$

$0.02[0.00 ; 0.27] 100.0 \%$

\section{Charadriiformes}

\section{Study}

Araujo, 2003

Sanmartin, 1973

Araujo, 2012

\section{Random effects model}

Heterogeneity: $I^{2}=61 \%, \tau^{2}=0.0045, p=0.08$

\section{Events Total}

$\begin{array}{rr}66 & 541 \\ 0 & 9 \\ 5 & 91\end{array}$

641

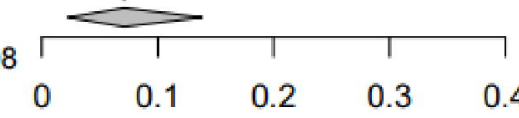

$\begin{array}{lllll}0 & 0.1 & 0.2 & 0.3 & 0.4\end{array}$

Proportion

$95 \%-\mathrm{Cl}$ Weight

$0.12[0.10 ; 0.15] 54.2 \%$ $0.00[0.00 ; 0.34] \quad 8.7 \%$ $0.05[0.02 ; 0.12] \quad 37.1 \%$

$0.07[0.02 ; 0.14] 100.0 \%$ 